\title{
Invariant K-minimal Sets in the Discrete and Continuous Settings
}

\author{
Natan Kruglyak and Eric Setterqvist \\ Journal Article
}

\section{Tweet}

N.B.: When citing this work, cite the original article.

This is a copy of the original publication which is available at www.springerlink.com:

Natan Kruglyak and Eric Setterqvist, Invariant K-minimal Sets in the Discrete and Continuous Settings, Journal of Fourier Analysis and Applications, 2016, pp.1-40.

http://dx.doi.org/10.1007/s00041-016-9479-5

Copyright: Springer Verlag (Germany)

http://www.springerlink.com/?MUD=MP

Postprint available at: Linköping University Electronic Press

http://urn.kb.se/resolve?urn=urn:nbn:se:liu:diva-132425

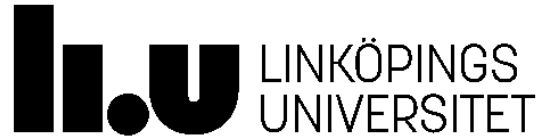




\title{
INVARIANT $K$-MINIMAL SETS IN THE DISCRETE AND CONTINUOUS SETTINGS
}

\author{
NATAN KRUGLYAK AND ERIC SETTERQVIST
}

\begin{abstract}
A sufficient condition for a set $\Omega \subset L^{1}\left([0,1]^{m}\right)$ to be invariant $K$ minimal with respect to the couple $\left(L^{1}\left([0,1]^{m}\right), L^{\infty}\left([0,1]^{m}\right)\right)$ is established. Through this condition, different examples of invariant $K$-minimal sets are constructed. In particular, it is shown that the $L^{1}$-closure of the image of the $L^{\infty}$-ball of smooth vector fields with support in $(0,1)^{m}$ under the divergence operator is an invariant $K$-minimal set.

The constructed examples have finite-dimensional analogues in terms of invariant $K$-minimal sets with respect to the couple $\left(\ell^{1}, \ell^{\infty}\right)$ on $\mathbb{R}^{n}$. These finite-dimensional analogues are interesting in themselves and connected to applications where the element with minimal $K$-functional is important. We provide a convergent algorithm for computing the element with minimal $K$ functional in these and other finite-dimensional invariant $K$-minimal sets.
\end{abstract}

\section{INTRODUCTION}

The importance of the quantity

$$
f^{* *}(t):=\frac{1}{t} \int_{0}^{t} f^{*}(s) d s
$$

where $f^{*}$ denotes the decreasing rearrangement of $f \in L^{1}+L^{\infty}$, in harmonic analysis is well-known. A celebrated result connecting this quantity with the couple $\left(L^{1}, L^{\infty}\right)$ is the following characterization established by A. P. Calderón in [5]: given $f, g \in L^{1}+L^{\infty}$, the condition

$$
g^{* *} \leq f^{* *}
$$

is equivalent to the existence of a linear operator $T: L^{1}+L^{\infty} \rightarrow L^{1}+L^{\infty}$ with $\|T\|_{L^{1} \rightarrow L^{1}} \leq 1$ and $\|T\|_{L^{\infty} \rightarrow L^{\infty}} \leq 1$ such that $T f=g$.

In the present paper we will provide a general construction of closed and convex subsets $\Omega$ of $L^{1}\left([0,1]^{m}\right)$ where for any given $f \in L^{1}\left([0,1]^{m}\right)$, there is an element $g_{f} \in \Omega$ such that

$$
\left(g_{f}-f\right)^{* *} \leq(g-f)^{* *}
$$

holds for all $g \in \Omega$. A simple example of such set is the $L^{\infty}$-ball of radius $t>0$, see Corollary [5.1, However, there exist much more complicated examples.

The inequality (1) can equivalently be expressed in terms of Peetre's $K$-functional for the couple $\left(L^{1}, L^{\infty}\right)$, more precisely the element $g_{f}$ satisfies

$$
K\left(t, g_{f}-f ; L^{1}, L^{\infty}\right)=\int_{0}^{t}\left(g_{f}-f\right)^{*} d s \leq \int_{0}^{t}(g-f)^{*} d s=K\left(t, g-f ; L^{1}, L^{\infty}\right)
$$

2010 Mathematics Subject Classification. Primary 46E30 Secondary 46N10.

Key words and phrases. Invariant $K$-minimal sets, taut strings, real interpolation. 
for all $g \in \Omega$ and every $t>0$. Recall that for a general Banach couple $\left(X_{0}, X_{1}\right)$, the $K$-functional is given by

$$
K\left(t, x ; X_{0}, X_{1}\right):=\inf _{x=x_{0}+x_{1}}\left(\left\|x_{0}\right\|_{X_{0}}+t\left\|x_{1}\right\|_{X_{1}}\right)
$$

for $x \in X_{0}+X_{1}$ and $t>0$. We refer to [2] or [4] for an introduction to the theory of real interpolation.

To put our results in a general framework, we introduce the notion of invariant $K$-minimal sets:

Definition 1.1. Given a Banach couple $\left(X_{0}, X_{1}\right)$, a set $\Omega \subset X_{0}+X_{1}$ is called invariant $K$-minimal with respect to $\left(X_{0}, X_{1}\right)$ if for every $a \in X_{0}+X_{1}$ there exists an element $x_{*, a} \in \Omega$ such that

$$
K\left(t, x_{*, a}-a ; X_{0}, X_{1}\right) \leq K\left(t, x-a ; X_{0}, X_{1}\right)
$$

holds for all $x \in \Omega$ and every $t>0$.

From Definition 1.1 it follows that $x_{*, a}$ is the nearest element of $a$ in $\Omega$ with respect to the norms of all exact interpolation spaces of $\left(X_{0}, X_{1}\right)$ generated by the $K$-method of the theory of real interpolation. In particular, $x_{*, a}$ is the nearest element of $a$ in $\Omega$ with respect to the norms of all interpolation spaces $\left(X_{0}, X_{1}\right)_{\theta, q}$, $0<\theta<1,1 \leq q \leq \infty$. As an example, consider the specific couple $\left(X_{0}, X_{1}\right) \stackrel{=}{=}$ $\left(L^{1}, L^{\infty}\right)$. Then the element $x_{*, a}$ is the nearest element of $a$ in $\Omega$ with respect to the norms of all $L^{p}$-spaces, $1<p<\infty$, i.e. element of best approximation of $a$ in $\Omega$ exists and is invariant with respect to all $L^{p}$-norms, $1<p<\infty$.

Note further from Definition 1.1 that if the set $\Omega \subset X_{0}+X_{1}$ is invariant $K$ minimal, then the set $\Omega+a$, for any $a \in X_{0}+X_{1}$, is also invariant $K$-minimal. This is the reason for calling the set invariant $K$-minimal.

Our motivation for introducing invariant $K$-minimal sets was the taut string problem considered by Dantzig, see [7, in connection with problems in optimal control. Taut string problems have since then appeared in a broad range of applications including statistics, see [1] and [15], image processing, see [19], stochastic processes, see [13], and communication theory, see [18] and [20]. A brief presentation of the taut string problem is given in Section 2.2

Now, let us recall some notion and results from [12].

Definition 1.2. The vectors

$$
v=\left\{\begin{array}{l} 
\pm e_{k}, k \in\{1, \ldots, n\}, \\
\pm\left(e_{k}+e_{l}\right), k, l \in\{1, \ldots, n\}, \quad k \neq l, \\
\pm\left(e_{k}-e_{l}\right), \quad k, l \in\{1, \ldots, n\}, k \neq l,
\end{array}\right.
$$

where $\left\{e_{i}\right\}_{i=1}^{n}$ is the standard basis of $\mathbb{R}^{n}$, will be referred to as special directions in $\mathbb{R}^{n}$.

Definition 1.3 (Special cone property). Let $\Omega \subset \mathbb{R}^{n}$ be closed and convex. For $x \in \Omega$, take all special directions $v$ such that $x+\beta v \in \Omega$ for sufficiently small $\beta>0$. Let $S_{x}$ denote the set of all such special directions at the element $x \in \Omega$. Further, let $K_{x}=\left\{y \in \mathbb{R}^{n}: y=\sum_{v \in S_{x}} \alpha_{v} v, \alpha_{v} \geq 0\right\}$ be the convex cone generated by the special directions in $S_{x}$. We say that $\Omega$ has the special cone property if $\left(x+K_{x}\right) \cap \Omega=\Omega$ for each $x \in \Omega$. 
The main result of 12 is the following characterization of all bounded, closed and convex sets in $\mathbb{R}^{n}$ that are invariant $K$-minimal with respect to the couple $\left(\ell^{1}, \ell^{\infty}\right)$ on $\mathbb{R}^{n}$ :

Theorem 1.1. A bounded, closed and convex set $\Omega \subset \mathbb{R}^{n}$ is invariant $K$-minimal with respect to the couple $\left(\ell^{1}, \ell^{\infty}\right)$ on $\mathbb{R}^{n}$ if and only if it has the special cone property.

What we will use in the sequel is the following sufficient condition which also was established in [12:

Theorem 1.2. Let $\Omega \subset \mathbb{R}^{n}$ be a closed and convex set that has the special cone property. Then $\Omega$ is an invariant $K$-minimal set with respect to $\left(\ell^{1}, \ell^{\infty}\right)$ on $\mathbb{R}^{n}$.

Note that the set $\Omega$ in Theorem 1.2 needs not to be bounded.

Besides the introduction, the paper is organized into four sections. Section 2 contains examples of invariant $K$-minimal sets with respect to $\left(\ell^{1}, \ell^{\infty}\right)$ on $\mathbb{R}^{n}$. In Section 3, an algorithm for computing the element of minimal $K$-functional is provided for sets satisfying the special cone property. Further, Section 4 contains a sufficient condition for a set $\Omega \subset L^{1}\left([0,1]^{m}\right)$ to be invariant $K$-minimal with respect to the couple $\left(L^{1}\left([0,1]^{m}\right), L^{\infty}\left([0,1]^{m}\right)\right)$. Finally, in Section 5 examples of invariant $K$-minimal sets with respect to $\left(L^{1}\left([0,1]^{m}\right), L^{\infty}\left([0,1]^{m}\right)\right)$ are provided. Below we will briefly describe some results from these sections.

Applying Theorem 1.2, we give concrete examples of invariant $K$-minimal sets with respect to $\left(\ell^{1}, \ell^{\infty}\right)$ on $\mathbb{R}^{n}$ in Section 2 of the paper. The given examples are used in the proof of the results in the continuous setting in Section 5. Moreover, these examples are of independent interest and important for applications. The most interesting example, in our opinion, is the following. Let $(V, E)$ be a finite directed graph with number of vertices $|V|=n$ and number of edges $|E|=m$. By $S_{V}$ we denote the $n$-dimensional space of real-valued functions defined on $V$. Analogously, $S_{E}$ denotes the $m$-dimensional space of real-valued functions defined on $E$. The divergence operator div $: S_{E} \rightarrow S_{V}$ is defined by

$$
(\operatorname{div} f)\left(v_{k}\right)=\sum_{i:\left(v_{i}, v_{k}\right) \in E} f\left(v_{i}, v_{k}\right)-\sum_{j:\left(v_{k}, v_{j}\right) \in E} f\left(v_{k}, v_{j}\right) .
$$

The elements of $S_{E}$ can be thought of as flows on the graph $(V, E)$ which gives the interpretation of the divergence at a vertex as the sum of all incoming flows minus the sum of all outgoing flows.

Given $F, G \in S_{E}$ with $F \leq G$, i.e. $F\left(\left(v_{i}, v_{k}\right)\right) \leq G\left(\left(v_{i}, v_{k}\right)\right)$ for every $\left(v_{i}, v_{k}\right) \in$ $E$, consider the rectangular domain $R_{F, G}=\left\{f \in S_{E}: F \leq f \leq G\right\}$ in $S_{E}$. The following result holds:

Theorem 1.3. The set $\operatorname{div}\left(R_{F, G}\right)$, i.e. the image of $R_{F, G}$ under the divergence operator div, is an invariant $K$-minimal set with respect to $\left(\ell^{1}, \ell^{\infty}\right)$ on $S_{V}$.

Thinking of the elements of $S_{E}$ as flows on the graph $(V, E)$, Theorem 1.3 can be seen as a result on optimal network flows. This result seems to have many potential applications. A brief discussion concerning applications is given after Remark 2.5. In connection with Theorem 1.3, we would like to mention the interesting paper 21] where a similar result is formulated, see Theorem 1 on p. 552. Unfortunately, a complete proof of that result has not been published. 
An important aspect in applications is how to construct the nearest element $x_{*, a}$ to $a$. In Section 3 we present an algorithm for computing $x_{*, a} \in \Omega$ where $\Omega$ is a closed and convex set in $\mathbb{R}^{n}$ with the special cone property. The algorithm is constructed through iterative projections of a starting element $x \in \Omega$ along special directions. We prove that the algorithm converges, for any given starting element $x \in \Omega$, towards $x_{*, a}$.

In Section 4 we turn to the continuous setting and consider invariant $K$-minimal sets with respect to the couple $\left(L^{1}\left([0,1]^{m}\right), L^{\infty}\left([0,1]^{m}\right)\right), m \in \mathbb{N}$. Note that in the paper real $L^{p}$-spaces are considered. We present a sufficient condition for a set $\Omega \subset$ $L^{1}\left([0,1]^{m}\right)$ to be invariant $K$-minimal with respect to $\left(L^{1}\left([0,1]^{m}\right), L^{\infty}\left([0,1]^{m}\right)\right)$. To briefly describe the result, let us introduce the averaging operators $P_{n}, n \in \mathbb{N}$. For a real-valued Lebesgue measurable function $f$ on $[0,1]^{m}$ we define

$$
P_{n} f:=\sum_{i_{1}, \ldots, i_{m}=1}^{n} \frac{1}{\left(\frac{1}{n}\right)^{m}}\left(\int_{\Gamma_{i_{1} \ldots i_{m}}^{n}} f(s) d s\right) \chi_{\Gamma_{i_{1} \ldots i_{m}}^{n}}
$$

where $\Gamma_{i_{1} \ldots i_{m}}^{n}:=\left[\frac{i_{1}-1}{n}, \frac{i_{1}}{n}\right) \times \ldots \times\left[\frac{i_{m}-1}{n}, \frac{i_{m}}{n}\right), i_{1}, \ldots, i_{m}=1, \ldots, n$, and $\chi_{\Gamma_{i_{1} \ldots i_{m}}^{n}}$ is the characteristic function of $\Gamma_{i_{1} \ldots i_{m}}^{n}$. Next, let $S_{n}\left([0,1]^{m}\right)$ denote the $n^{m}$-dimensional space of piecewise constant functions on $[0,1]^{m}$ given by

$$
S_{n}\left([0,1]^{m}\right)=\left\{g: g=\sum_{i_{1}, \ldots, i_{m}=1}^{n} c_{i_{1} \ldots i_{m}} \chi_{\Gamma_{i_{1} \ldots i_{m}}^{n}}, c_{i_{1} \ldots i_{m}} \in \mathbb{R}\right\} .
$$

For each $g=\sum_{i_{1}, \ldots, i_{m}=1}^{n} c_{i_{1} \ldots i_{m}} \chi_{\Gamma_{i_{1} \ldots i_{m}}^{n}} \in S_{n}\left([0,1]^{m}\right)$, we have a corresponding uniquely determined coefficient vector $c=\left(c_{1 \ldots 1}, \ldots, c_{n \ldots n}\right) \in \mathbb{R}^{n^{m}}$. Given $\Gamma \subset$ $S_{n}\left([0,1]^{m}\right)$, we denote by $\widetilde{\Gamma}$ the set in $\mathbb{R}^{n^{m}}$ which consists of all coefficient vectors corresponding to the elements in $\Gamma$. With this notation introduced, we can now formulate the result:

Theorem 1.4. Let $\left\{\Omega_{2^{n}}\right\}_{n \in \mathbb{N}}$ be a family of sets such that for each $n \in \mathbb{N}$ (i) $\Omega_{2^{n}}$ is a closed and convex subset of $S_{2^{n}}\left([0,1]^{m}\right)$, (ii) $P_{2^{n}}: \Omega_{2^{n+1}} \rightarrow \Omega_{2^{n}}$, and (iii) the set of coefficient vectors $\widetilde{\Omega}_{2^{n}}$ is invariant $K$-minimal with respect to $\left(\ell^{1}, \ell^{\infty}\right)$ on $\mathbb{R}^{2^{n m}}$. Then the set

$$
\Omega:=\left\{h \in L^{1}\left([0,1]^{m}\right): P_{2^{n}} h \in \Omega_{2^{n}}, \forall n \in \mathbb{N}\right\}
$$

is invariant $K$-minimal with respect to $\left(L^{1}\left([0,1]^{m}\right), L^{\infty}\left([0,1]^{m}\right)\right)$.

The proof of this theorem uses uniform boundedness in $L^{1}$ and uniform integrability combined with Mazur's lemma.

Finally, in Section [5 we construct, through Theorem 1.4 concrete examples of invariant $K$-minimal sets with respect to $\left(L^{1}\left([0,1]^{m}\right), L^{\infty}\left([0,1]^{m}\right)\right)$. One of the results in this section is an infinite-dimensional analogue of Theorem 1.3. This result was inspired by the remarkable Theorem 4.46 in [19, p. 148]. Given $f \in$ $L^{2}\left((0,1)^{m}\right)$, their result implies the existence of an element $g_{f} \in L^{2}\left((0,1)^{m}\right)$, satisfying $\left\|g_{f}-f\right\|_{*} \leq 1$, such that

$$
\int_{(0,1)^{m}} \varphi\left(g_{f}(x)\right) d x=\inf _{\substack{g \in L^{2}\left((0,1)^{m}\right) \\\|g-f\|_{*} \leq 1}} \int_{(0,1)^{m}} \varphi(g(x)) d x
$$


holds for all convex functions $\varphi: \mathbb{R} \rightarrow \mathbb{R}$. Here $\|\cdot\|_{*}$ denotes the $*$-number which is closely related to the $G$-norm introduced by Y. Meyer in [16] in connection with investigations of the Rudin-Osher-Fatemi image denoising model.

Before giving the infinite-dimensional analogue of Theorem 1.3, we need some notation. Let $C_{c}^{\infty}\left((0,1)^{m}, \mathbb{R}^{m}\right)$ denote the space of $m$-dimensional smooth vector fields with support in $(0,1)^{m}$. We define $L^{\infty}\left([0,1]^{m}, \mathbb{R}^{m}\right)$ as the space of all $m$ dimensional Lebesgue measurable vector fields $\mathbf{F}=\left(F_{1}, \ldots, F_{m}\right):[0,1]^{m} \rightarrow \mathbb{R}^{m}$ such that $\|\mathbf{F}\|_{L^{\infty}}:=\max _{1 \leq i \leq m}\left\{\left\|F_{i}\right\|_{L^{\infty}}\right\}<\infty$. Now, the following infinite-dimensional analogue of Theorem 1.3 holds:

Theorem 1.5. Let

$$
M:=\left\{\mathbf{F} \in C_{c}^{\infty}\left((0,1)^{m}, \mathbb{R}^{m}\right):\|\mathbf{F}\|_{L^{\infty}} \leq 1\right\}
$$

and

$$
\operatorname{div} M:=\left\{f \in C_{c}^{\infty}\left((0,1)^{m}\right): f=\operatorname{div} \mathbf{F}, \mathbf{F} \in M\right\} .
$$

Then the closure of $\operatorname{div} M$ in $L^{1}$ is an invariant $K$-minimal set with respect to $\left(L^{1}\left([0,1]^{m}\right), L^{\infty}\left([0,1]^{m}\right)\right)$.

\section{Finite-dimensional EXAmples}

In this section we present examples of invariant $K$-minimal sets with respect to $\left(\ell^{1}, \ell^{\infty}\right)$ on $\mathbb{R}^{n}$. These examples have interesting applications and also serve as prototypes for constructing invariant $K$-minimal sets with respect to $\left(L^{1}\left([0,1]^{m}\right), L^{\infty}\left([0,1]^{m}\right)\right), m \in \mathbb{N}$.

Before proceeding to the examples we recall the notion of invariant $\varphi$-minimal sets which are closely related to invariant $K$-minimal sets with respect to $\left(\ell^{1}, \ell^{\infty}\right)$.

Definition 2.1. A set $\Omega \subset \mathbb{R}^{n}$ will be called invariant $\varphi$-minimal if for every $a \in \mathbb{R}^{n}$ there exists an element $x_{*, a} \in \Omega$ such that

$$
\sum_{i=1}^{n} \varphi\left(x_{*, a, i}-a_{i}\right) \leq \sum_{i=1}^{n} \varphi\left(x_{i}-a_{i}\right), \forall x \in \Omega
$$

for all convex functions $\varphi: \mathbb{R} \rightarrow \mathbb{R}$.

As noted in [12, Remark 3.1], we have the following counterpart of Theorem 1.2 for invariant $\varphi$-minimal sets:

Theorem 2.1. Let $\Omega \subset \mathbb{R}^{n}$ be a closed and convex set that satisfies the special cone property. Moreover, assume that $\sum_{i=1}^{n} x_{i}=C$, where $C$ is some fixed real number, for every $x \in \Omega$. Then $\Omega$ is an invariant $\varphi$-minimal set.

We will use notation from Definitions 1.2 and 1.3 frequently in the sequel of this section.

2.1. Rectangular domains. Our first example of invariant $K$-minimal sets with respect to $\left(\ell^{1}, \ell^{\infty}\right)$ are rectangular domains.

Proposition 2.1. Given $F, G \in \mathbb{R}^{n}$ with $F_{i} \leq G_{i}, i=1, \ldots, n$, let

$$
\Omega:=\left\{u \in \mathbb{R}^{n}: F_{i} \leq u_{i} \leq G_{i}\right\} .
$$

Then $\Omega$ is an invariant $K$-minimal set with respect to $\left(\ell^{1}, \ell^{\infty}\right)$ on $\mathbb{R}^{n}$. 
Proof. It is straightforward to show that $\Omega$ has the special cone property by considering the special directions $e_{i}, i=1, \ldots, n$. By applying Theorem 1.2, the result then follows.

2.2. Taut string problem. We now recall the taut string problem. Let $a=x_{0}<$ $x_{1}<\ldots<x_{n}=b$ be $n+1$ points on the real line, $n \geq 1$, given by

$$
x_{i}=a+\frac{i(b-a)}{n}, i=0,1, \ldots, n .
$$

Consider two continuous functions $F \leq G$ on the interval $[a, b]$ that are linear on the intervals $\left[a, x_{1}\right],\left[x_{1}, x_{2}\right], \ldots,\left[x_{n-1}, b\right]$. Let $\Gamma_{F, G}$ denote the set of all continuous piecewise linear functions $f$ on $[a, b] \subset \mathbb{R}$ with nodes in $x_{i}, i=0,1, \ldots, n$, and which satisfy the inequalities $F \leq f \leq G$.

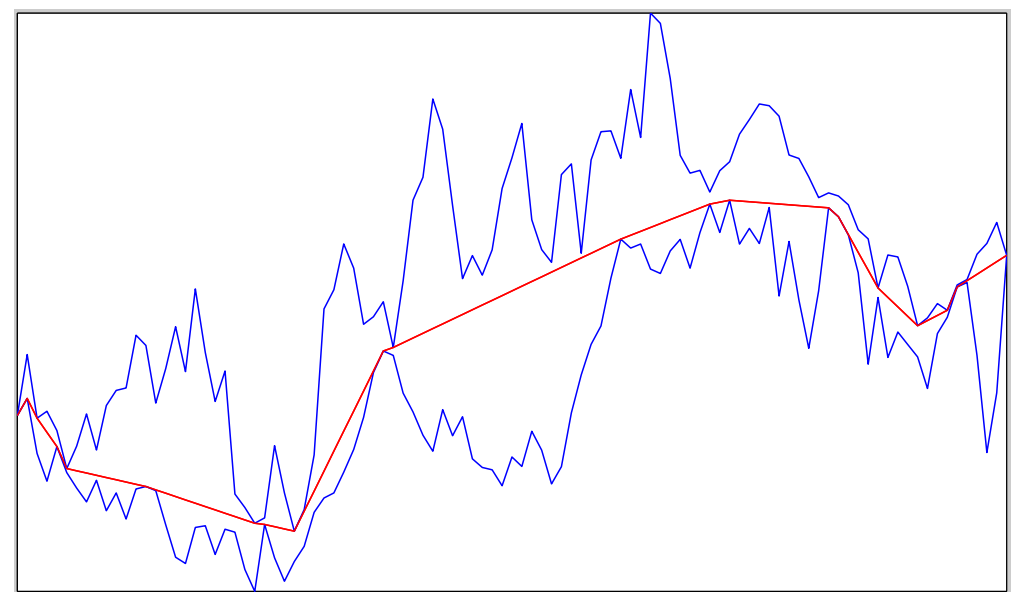

FiguRE 1. Taut string (red) inside the corridor $\Gamma_{F, G}$.

The taut string problem can be formulated as:

Problem 2.1. Find the function $f_{*} \in \Gamma_{F, G}$ that satisfies

$$
\int_{a}^{b} \sqrt{1+\left(f_{*}^{\prime}(x)\right)^{2}} d x=\inf _{f \in \Gamma_{F, G}} \int_{a}^{b} \sqrt{1+\left(f^{\prime}(x)\right)^{2}} d x .
$$

Remark 2.1. Classically, the functions $F$ and $G$ are assumed in addition to satisfy $F(a)=G(a)$ and $F(b)=G(b)$. However, these assumptions are not necessary for Theorem 2.2 below.

The connection between the taut string problem and invariant $K$-minimal sets is given by:

Theorem 2.2. The set

$$
\Omega:=\left\{u \in \mathbb{R}^{n}: u_{i}=\frac{f\left(x_{i}\right)-f\left(x_{i-1}\right)}{x_{i}-x_{i-1}}, i=1, \ldots, n, f \in \Gamma_{F, G}\right\}
$$

is an invariant $K$-minimal set with respect to $\left(\ell^{1}, \ell^{\infty}\right)$ on $\mathbb{R}^{n}$. 
Remark 2.2. Note that the set $\Omega$ can be interpreted as the set of piecewise constant derivatives of the functions in $\Gamma_{F, G}$. Theorem 2.2 was formulated in [12]. As the taut string problem has many applications and the proof of Theorem [2.2 in [12] was rather sketchy, we provide a proof in full detail here.

Proof. Note that $\Omega$ is closed and convex. The next step is to show that $\Omega$ has the special cone property. Applying Theorem 1.2 will then give the result.

The definition of $\Omega$ and recalling that $x_{i}-x_{i-1}=(b-a) / n, i=1, \ldots, n$, imply that $u \in \Omega$ can be represented according to

$$
\begin{aligned}
u= & \sum_{i=1}^{n}\left(\frac{f\left(x_{i}\right)-f\left(x_{i-1}\right)}{x_{i}-x_{i-1}}\right) e_{i}= \\
& =\frac{f\left(x_{0}\right)}{(b-a) / n}\left(-e_{1}\right)+\sum_{i=1}^{n-1} \frac{f\left(x_{i}\right)}{(b-a) / n}\left(e_{i}-e_{i+1}\right)+\frac{f\left(x_{n}\right)}{(b-a) / n} e_{n}
\end{aligned}
$$

for some $f \in R_{F, G}$. Given $u$, we now take an arbitrary element $v \in \Omega \backslash\{u\}$ and show that $v \in u+K_{u}$. The element $v$ can be represented as

$$
\begin{aligned}
v= & \sum_{i=1}^{n}\left(\frac{g\left(x_{i}\right)-g\left(x_{i-1}\right)}{x_{i}-x_{i-1}}\right) e_{i}= \\
& =\frac{g\left(x_{0}\right)}{(b-a) / n}\left(-e_{1}\right)+\sum_{i=1}^{n-1} \frac{g\left(x_{i}\right)}{(b-a) / n}\left(e_{i}-e_{i+1}\right)+\frac{g\left(x_{n}\right)}{(b-a) / n} e_{n}
\end{aligned}
$$

where $g \in R_{F, G}$. We can then express

$$
\begin{aligned}
& v-u= \\
& =\frac{g\left(x_{0}\right)-f\left(x_{0}\right)}{(b-a) / n}\left(-e_{1}\right)+\sum_{i=1}^{n-1} \frac{g\left(x_{i}\right)-f\left(x_{i}\right)}{(b-a) / n}\left(e_{i}-e_{i+1}\right)+\frac{g\left(x_{n}\right)-f\left(x_{n}\right)}{(b-a) / n} e_{n} .
\end{aligned}
$$

Let us consider how the signs of the coefficients $\frac{g\left(x_{i}\right)-f\left(x_{i}\right)}{(b-a) / n}$ in (2) imply the inclusion of certain special directions in $S_{u}$.

(i) Consider first $i=0$. If $g\left(x_{0}\right)>f\left(x_{0}\right)$ then $f\left(x_{0}\right)<G\left(x_{0}\right)$ which implies $u+\beta\left(-e_{1}\right) \in \Omega$ for small enough $\beta>0$. Hence, $-e_{1} \in S_{u}$ in this case. On the other hand, if $g\left(x_{0}\right)<f\left(x_{0}\right)$ then $F\left(x_{0}\right)<f\left(x_{0}\right)$ which implies $e_{1} \in S_{u}$.

(ii) Consider next $i=n$. If $g\left(x_{n}\right)>f\left(x_{n}\right)$ then $f\left(x_{n}\right)<G\left(x_{n}\right)$ which implies $u+\beta e_{n} \in \Omega$ for small enough $\beta>0$. Hence, $e_{n} \in S_{u}$ in this case. On the other hand, if $g\left(x_{n}\right)<f\left(x_{n}\right)$ then $F\left(x_{n}\right)<f\left(x_{n}\right)$ which implies $-e_{n} \in S_{u}$.

(iii) Finally, consider $i \in\{1, \ldots, n-1\}$. If $g\left(x_{i}\right)>f\left(x_{i}\right)$ then $f\left(x_{i}\right)<G\left(x_{i}\right)$ which implies $u+\beta\left(e_{i}-e_{i+1}\right) \in \Omega$ for small enough $\beta>0$. Hence, $e_{i}-e_{i+1} \in S_{u}$ in this case. On the other hand, if $g\left(x_{i}\right)<f\left(x_{i}\right)$ then $F\left(x_{i}\right)<f\left(x_{i}\right)$ which implies $-\left(e_{i}-e_{i+1}\right) \in S_{u}$.

Taken together, (i)-(iii) and (2) give that $v$ can be expressed as a sum of $u$ and positively scaled special directions in $S_{u}$, i.e. $v \in u+K_{u}$. As $v \in \Omega \backslash\{u\}$ was arbitrarily chosen, it follows that $\Omega \subset u+K_{u}$ or equivalently $\Omega=\left(u+K_{u}\right) \cap \Omega$. Recalling that $u \in \Omega$ also was arbitrarily chosen from the beginning, we then conclude that $\Omega$ has the special cone property.

Applying Theorem 1.2 finally gives that $\Omega$ is an invariant $K$-minimal set with respect to $\left(\ell^{1}, \ell^{\infty}\right)$ on $\mathbb{R}^{n}$. 
Remark 2.3. From the proof of Theorem 2.2 we know that $\Omega$ is a closed and convex set that has the special cone property. If $F(a)=G(a)$ and $F(b)=G(b)$, then for any $u \in \Omega$ we have

$$
\sum_{i=1}^{n} u_{i}=\frac{f\left(x_{n}\right)-f\left(x_{0}\right)}{(b-a) / n}=\frac{F\left(x_{n}\right)-F\left(x_{0}\right)}{(b-a) / n} .
$$

Theorem 2.1 therefore gives that $\Omega$ in this situation in addition is invariant $\varphi$ minimal.

2.3. Rectangular domain intersected with hyperplane. Our next example of invariant $K$-minimal sets concerns the intersection of a hyperplane of the type $\left\{u \in \mathbb{R}^{n}: \sum_{i=1}^{n} u_{i}=C\right\}$ with a rectangular domain. Sets of such type are considered in [1] in connection with optimization problems over partitions of a finite set.

We have the following result:

Theorem 2.3. Given $F, G \in \mathbb{R}^{n}$ with $F_{i} \leq G_{i}, i=1, \ldots, n$, let

$$
\Omega:=\left\{u \in \mathbb{R}^{n}: F_{i} \leq u_{i} \leq G_{i}, \sum_{i=1}^{n} u_{i}=C\right\}
$$

where the constant $C \in \mathbb{R}$ is such that $\sum_{i=1}^{n} F_{i} \leq C \leq \sum_{i=1}^{n} G_{i}$. Then $\Omega$ is an invariant $K$-minimal set with respect to $\left(\ell^{1}, \ell^{\infty}\right)$ on $\mathbb{R}^{n}$.

Proof. First note that $\Omega$ is closed and convex. We now show that $\Omega$ has the special cone property which then, by applying Theorem 1.2, will give the result.

Given $u \in \Omega$, let us show that certain special directions of the type $e_{i}-e_{j}$ are in the set $S_{u}$. Fix $i \in\{1, \ldots, n\}$ with $F_{i}<G_{i}$ and consider all $j \in\{1, \ldots, n\} \backslash\{i\}$. We have three cases.

(i) Suppose $u_{i}=F_{i}<G_{i}$. If $F_{j}<u_{j} \leq G_{j}$ then it is clear that $u+\beta\left(e_{i}-e_{j}\right) \in \Omega$ for small enough $\beta>0$, i.e. $e_{i}-e_{j} \in S_{u}$.

(ii) Suppose $F_{i}<u_{i}<G_{i}$. Here we have three different subcases: (a) If $u_{j}=$ $F_{j}<G_{j}$ then $u+\beta\left(-e_{i}+e_{j}\right) \in \Omega$ for small enough $\beta>0$, i.e. $-e_{i}+e_{j} \in S_{u}$. (b) If $F_{j}<G_{j}=u_{j}$ then $u+\beta\left(e_{i}-e_{j}\right) \in \Omega$ for small enough $\beta>0$, i.e. $e_{i}-e_{j} \in S_{u}$. (c) If $F_{j}<u_{j}<G_{j}$ then $u+\beta\left( \pm\left(e_{i}-e_{j}\right)\right) \in \Omega$ for small enough $\beta>0$, i.e. $\pm\left(e_{i}-e_{j}\right) \in S_{u}$.

(iii) Suppose $F_{i}<G_{i}=u_{i}$. If $F_{i} \leq u_{j}<G_{j}$ then $u+\beta\left(-e_{i}+e_{j}\right) \in \Omega$ for small enough $\beta>0$, i.e. $-e_{i}+e_{j} \in S_{u}$.

With the inclusion of special directions in $S_{u}$ according to (i)-(iii) at hand, let us now show $\left(u+K_{u}\right) \cap \Omega=\Omega$. Let $v \in \Omega \backslash\{u\}$ be given. Take an index $i_{1} \in\{1, \ldots, n\}$ that satisfies

$$
\left|v_{i_{1}}-u_{i_{1}}\right|=\min _{i \in\{1, \ldots, n\}: v_{i} \neq u_{i}}\left|v_{i}-u_{i}\right| .
$$

Next, take an index $j_{1} \in\{1, \ldots, n\}$ that satisfies

$$
\operatorname{sgn}\left(v_{j_{1}}-u_{j_{1}}\right)=-\operatorname{sgn}\left(v_{i_{1}}-u_{i_{1}}\right)
$$

where

$$
\operatorname{sgn}(x):=\left\{\begin{array}{l}
-1, x<0 \\
0, x=0 \\
1, x>0
\end{array}\right.
$$


Note that such index $j_{1}$ must exist since $\sum_{i=1}^{n} v_{i}=\sum_{i=1}^{n} u_{i}=C$. Taking into account (i)-(iii), we then conclude $\operatorname{sgn}\left(v_{i_{1}}-u_{i_{1}}\right)\left(e_{i_{1}}-e_{j_{1}}\right) \in S_{u}$. Set

$$
u^{1}:=u+\left|v_{i_{1}}-u_{i_{1}}\right| \operatorname{sgn}\left(v_{i_{1}}-u_{i_{1}}\right)\left(e_{i_{1}}-e_{j_{1}}\right) .
$$

By construction, $u^{1} \in \Omega$ and $u_{i_{1}}^{1}=v_{i_{1}}$. Moreover, if $u_{j_{1}}^{1} \neq v_{j_{1}}$ then

$$
\operatorname{sgn}\left(v_{j_{1}}-u_{j_{1}}^{1}\right)=\operatorname{sgn}\left(v_{j_{1}}-u_{j_{1}}\right)
$$

Note that the number of components where $u^{1}$ and $v$ differ are strictly less than the corresponding number of components where $u$ and $v$ differ.

Suppose $v \neq u^{1}$. We then repeat the procedure for $u^{1}$. First, take an index $i_{2} \in\{1, \ldots, n\}$ that satisfies

$$
\left|v_{i_{2}}-u_{i_{2}}^{1}\right|=\min _{i \in\{1, \ldots, n\}: v_{i} \neq u_{i}^{1}}\left|v_{i}-u_{i}^{1}\right| \text {, }
$$

and then an index $j_{2} \in\{1, \ldots, n\}$ that satisfies

$$
\operatorname{sgn}\left(v_{j_{2}}-u_{j_{2}}^{1}\right)=-\operatorname{sgn}\left(v_{i_{2}}-u_{i_{2}}^{1}\right) .
$$

Note that from (3) it follows that $\operatorname{sgn}\left(v_{i_{2}}-u_{i_{2}}^{1}\right)\left(e_{i_{2}}-e_{j_{2}}\right) \in S_{u}$. Then construct $u^{2}$ according to

$$
\begin{aligned}
u^{2}: & =u^{1}+\left|v_{i_{2}}-u_{i_{2}}^{1}\right| \operatorname{sgn}\left(v_{i_{2}}-u_{i_{2}}^{1}\right)\left(e_{i_{2}}-e_{j_{2}}\right)= \\
& =u+\left|v_{i_{1}}-u_{i_{1}}\right| \operatorname{sgn}\left(v_{i_{1}}-u_{i_{1}}\right)\left(e_{i_{1}}-e_{j_{1}}\right)+ \\
& \left|v_{i_{2}}-u_{i_{2}}^{1}\right| \operatorname{sgn}\left(v_{i_{2}}-u_{i_{2}}^{1}\right)\left(e_{i_{2}}-e_{j_{2}}\right) .
\end{aligned}
$$

If $v \neq u^{2}$ then repeat the procedure for $u^{2}$ and so on until we reach

$$
v=u^{m}=u+\sum_{k=1}^{m}\left|v_{i_{k}}-u_{i_{k}}\right| \operatorname{sgn}\left(v_{i_{k}}-u_{i_{k}}\right)\left(e_{i_{k}}-e_{j_{k}}\right)
$$

for some integer $m \in\{1, \ldots, n-1\}$ where $\operatorname{sgn}\left(v_{i_{k}}-u_{i_{k}}\right)\left(e_{i_{k}}-e_{j_{k}}\right) \in S_{u}, k=$ $1 \ldots, m$. So, after adding a finite number of positively scaled elements in $S_{u}$ to $u$ we will obtain $v$. Hence, $\left(u+K_{u}\right) \cap \Omega=\Omega$ as $v \in \Omega \backslash\{u\}$ was chosen arbitrarily. As $u \in \Omega$ in turn was chosen arbitrarily, it follows that $\Omega$ has the special cone property.

Applying Theorem 1.2 finally gives that $\Omega$ is an invariant $K$-minimal set with respect to $\left(\ell^{1}, \ell^{\infty}\right)$ on $\mathbb{R}^{n}$.

Remark 2.4. From the proof of Theorem 2.3 we know that $\Omega$ is a closed and convex set that has the special cone property. As $\sum_{i=1}^{n} u_{i}=C$ for every $u \in \Omega$ it follows from Theorem 2.1 that $\Omega$ in addition is invariant $\varphi$-minimal. This was also obtained in [11, Theorem 4.3] by a different approach.

2.4. Divergence of flows on graphs. We next consider a problem that in some sense is a generalization of the one-dimensional taut string problem with fixed ends, i.e. $F(a)=G(a)$ and $F(b)=G(b)$. Let $(V, E)$ be a finite directed graph with $n$ vertices $v_{1}, \ldots, v_{n} \in V$ and $m$ edges $\left(v_{i_{j}}, v_{k_{j}}\right) \in E, j=1, \ldots, m$. The edge $\left(v_{i}, v_{k}\right)$ is interpreted as directed from $v_{i}$ to $v_{k}$. By $S_{V}$ we denote the $n$-dimensional space of real-valued functions defined on $V$. Analogously, $S_{E}$ denotes the $m$-dimensional space of real-valued functions defined on $E$. The divergence operator div $: S_{E} \rightarrow S_{V}$ is defined by

$$
(\operatorname{div} f)\left(v_{k}\right)=\sum_{i:\left(v_{i}, v_{k}\right) \in E} f\left(v_{i}, v_{k}\right)-\sum_{j:\left(v_{k}, v_{j}\right) \in E} f\left(v_{k}, v_{j}\right) .
$$


The elements of $S_{E}$ can be thought of as flows on the graph $(V, E)$ which gives the interpretation of the divergence at a vertex as the sum of all incoming flows minus the sum of all outgoing flows.

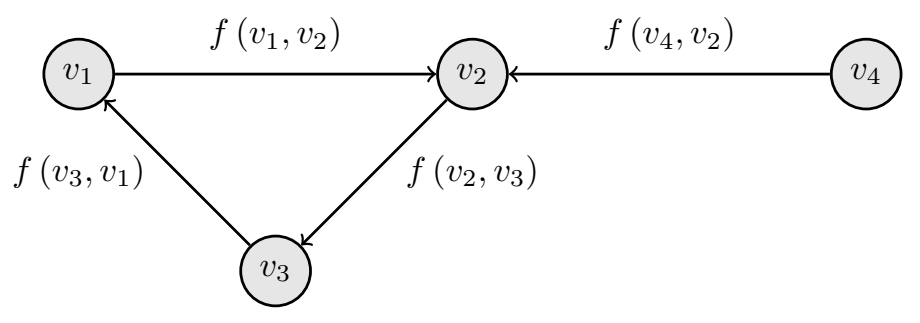

Figure 2. Example of a graph with flows.

Given $F, G \in S_{E}$ with $F \leq G$, i.e. $F\left(v_{i}, v_{k}\right) \leq G\left(v_{i}, v_{k}\right)$ for every $\left(v_{i}, v_{k}\right) \in E$, consider the rectangular domain $R_{F, G}$ in $S_{E}$ :

$$
R_{F, G}=\left\{f \in S_{E}: F \leq f \leq G\right\} .
$$

The following result holds

Theorem 2.4. The set $\operatorname{div}\left(R_{F, G}\right)$, i.e. the image of $R_{F, G}$ under the divergence operator div, is an invariant $K$-minimal set with respect to $\left(\ell^{1}, \ell^{\infty}\right)$ on $S_{V}$.

Proof. First, note that that we can identify $S_{E}$ with $\mathbb{R}^{m}$ and $S_{V}$ with $\mathbb{R}^{n}$. Below, we will consider $\operatorname{div}\left(R_{F, G}\right)$ as a subset of $\mathbb{R}^{n}$.

We note that $\operatorname{div}\left(R_{F, G}\right)$ is closed and convex. In order to prove the theorem, we will show that $\operatorname{div}\left(R_{F, G}\right)$ has the special cone property which, by applying Theorem 1.2, will give the result.

Take $u=\operatorname{div} f \in \operatorname{div}\left(R_{F, G}\right)$. As the flow term $f\left(v_{i_{j}}, v_{k_{j}}\right)$ is added in the term $(\operatorname{div} f)\left(v_{k_{j}}\right)$ and subtracted in the term $(\operatorname{div} f)\left(v_{i_{j}}\right)$ we can express $u$ according to

$$
u=\operatorname{div} f=\sum_{j=1}^{m} f\left(v_{i_{j}}, v_{k_{j}}\right)\left(e_{k_{j}}-e_{i_{j}}\right) .
$$

Take now $v=\operatorname{div} g \in \operatorname{div}\left(R_{F, G}\right) \backslash\{u\}$ and consider the difference $v-u$. In view of (5) we have

$$
v-u=\operatorname{div} g-\operatorname{div} f=\sum_{j=1}^{m}\left(g\left(v_{i_{j}}, v_{k_{j}}\right)-f\left(v_{i_{j}}, v_{k_{j}}\right)\right)\left(e_{k_{j}}-e_{i_{j}}\right) .
$$

Fix $j=1, \ldots, m$. We now show that certain special directions are in $S_{u}$.

(i) If $f\left(v_{i_{j}}, v_{k_{j}}\right)<g\left(v_{i_{j}}, v_{k_{j}}\right)$ then $f\left(v_{i_{j}}, v_{k_{j}}\right)<G\left(v_{i_{j}}, v_{k_{j}}\right)$. So, for small enough $\beta>0$ we have $f_{\beta} \in R_{F, G}$ where

$$
f_{\beta}(e):=\left\{\begin{array}{l}
f(e)+\beta, e=\left(v_{i_{j}}, v_{k_{j}}\right) \\
f(e), e \neq\left(v_{i_{j}}, v_{k_{j}}\right)
\end{array}\right.
$$

This implies $u+\beta\left(e_{k_{j}}-e_{i_{j}}\right) \in \Omega$ for small enough $\beta>0$. Hence, $e_{k_{j}}-e_{i_{j}} \in S_{u}$ in this case. 
(ii) On the other hand, if $g\left(v_{i_{j}}, v_{k_{j}}\right)<f\left(v_{i_{j}}, v_{k_{j}}\right)$ then $F\left(v_{i_{j}}, v_{k_{j}}\right)<f\left(v_{i_{j}}, v_{k_{j}}\right)$. So, for small enough $\beta>0$ we have $f_{\beta} \in R_{F, G}$ where

$$
f_{\beta}(e):=\left\{\begin{array}{l}
f(e)-\beta, e=\left(v_{i_{j}}, v_{k_{j}}\right) \\
f(e), e \neq\left(v_{i_{j}}, v_{k_{j}}\right)
\end{array}\right.
$$

This implies $u+\beta\left(-e_{k_{j}}+e_{i_{j}}\right) \in \Omega$ for small enough $\beta>0$. Hence, $-e_{k_{j}}+$ $e_{i_{j}} \in S_{u}$ in this case.

Taken together, (i)-(ii) and (6) give that $v$ can be expressed as a sum of $u$ and positively scaled special directions in $S_{u}$, i.e. $v \in u+K_{u}$. As $v \in \operatorname{div}\left(R_{F, G}\right) \backslash\{u\}$ was arbitrarily chosen, it follows that $\operatorname{div}\left(R_{F, G}\right) \subset u+K_{u}$ or equivalently $\operatorname{div}\left(R_{F, G}\right)=$ $\left(u+K_{u}\right) \cap \operatorname{div}\left(R_{F, G}\right)$. Recalling that $u \in \operatorname{div}\left(R_{F, G}\right)$ also was arbitrarily chosen from the beginning, we then conclude that $\operatorname{div}\left(R_{F, G}\right)$ has the special cone property.

Applying Theorem 1.2 finally gives that $\Omega$ is an invariant $K$-minimal set with respect to $\left(\ell^{1}, \ell^{\infty}\right)$ on $S_{V}$.

Remark 2.5. From the proof of Theorem 2.4 we know that $\Omega$ is a closed and convex set that has the special cone property. As $\sum_{v \in V} u(v)=0$ for every $u \in \operatorname{div}\left(R_{F, G}\right)$ it follows from Theorem 2.1 that $\operatorname{div}\left(R_{F, G}\right)$ in addition is invariant $\varphi$-minimal, i.e. given $a \in S_{V}$ there exists an element $x_{*, a} \in \operatorname{div}\left(R_{F, G}\right)$ such that

$$
\sum_{v \in V} \varphi\left(x_{*, a, v}-a_{v}\right) \leq \sum_{v \in V} \varphi\left(x_{v}-a_{v}\right)
$$

for every $x \in \operatorname{div}\left(R_{F, G}\right)$ and every convex function $\varphi: \mathbb{R} \rightarrow \mathbb{R}$.

We now briefly discuss a possible application of Theorem 2.4. Suppose the directed graph $(V, E)$ represents a network where water flows through pipes (edges) to different locations (vertices). Each pipe $e \in E$ has a given capacity interval $[0, G(e)], G(e) \geq 0$. Assume first that the network is running in normal condition and then one or several pipes becomes broken with reduced or no capacity. This might affect incoming and/or outgoing flow at some locations. Recall that the divergence at a location is the difference between incoming and outgoing flow. An increasing divergence at a location can be thought of as a flooding there whereas a decreasing divergence at a location can be thought of as a drought there. Let $G^{\prime}$ denote the upper capacity function of the broken network. Suppose the divergence in the network during normal conditions is given by $a \in S_{V}$. We modify the flows in the network such that the resulting divergence $x_{*, a} \in \operatorname{div}\left(R_{0, G^{\prime}}\right)$ satisfies

$$
\sum_{v \in V} \varphi\left(x_{*, a, v}-a_{v}\right)=\inf _{x \in \operatorname{div}\left(R_{0, G^{\prime}}\right)} \sum_{v \in V} \varphi\left(x_{v}-a_{v}\right)
$$

for all convex function $\varphi: \mathbb{R} \rightarrow \mathbb{R}$. This can be viewed as minimizing the total impact of the broken pipes in the network or in other words optimally adjust the flows to the new network conditions.

This approach of optimal management of a network under changing conditions is useful in diverse applications. For examples in communication theory and image processing, we refer to 20] respectively [17. We plan to apply this approach to more applications in future investigations. 


\section{An ALGORITHM}

Suppose $\Omega \subset \mathbb{R}^{n}$ is closed and convex and satisfies the special cone property (recall Definition 1.3). Then by Theorem 1.2 it follows that for given $a \in \mathbb{R}^{n}$, there exists an element $x_{*, a} \in \Omega$ such that

$$
K\left(t, x_{*, a}-a ; \ell^{1}, \ell^{\infty}\right) \leq K\left(t, x-a ; \ell^{1}, \ell^{\infty}\right)
$$

for every $x \in \Omega$ and $t>0$. In this section, we provide an algorithm that computes $x_{*, a}$. The algorithm is constructed through $\ell^{2}$-minimization along search directions given by the special directions. Before formulating the result of this section, we introduce some notation.

Fix $a, v \in \mathbb{R}^{n}$. Let $P_{a, v}: \Omega \rightarrow \Omega$ denote the operator defined by the conditions

$$
P_{a, v}(x) \in\{x+t v: t \in \mathbb{R}\} \cap \Omega
$$

and

$$
\left\|P_{a, v}(x)-a\right\|_{\ell^{2}}=\inf _{u \in\{x+t v: t \in \mathbb{R}\} \cap \Omega}\|u-a\|_{\ell^{2}} .
$$

So, $P_{a, v}$ maps $x \in \Omega$ onto the element of best approximation of $a$, with respect to $\ell^{2}$-norm, on the line segment $\{x+t v: t \in \mathbb{R}\} \cap \Omega$ with direction vector $v$.

As before, given $x \in \Omega$ let $S_{x}$ denote the set of all special directions $v$ such that $x+\beta v \in \Omega$ for small enough $\beta>0$. Choose a special direction $v_{x} \in S_{x}$ such that

$$
\left\|P_{a, v_{x}}(x)-a\right\|_{\ell^{2}}=\min _{v \in S_{x}}\left\|P_{a, v}(x)-a\right\|_{\ell^{2}} .
$$

If there are several special directions $v$ that fulfill (7) we can choose $v_{x}$ to be any of them. The operator $P_{a}: \Omega \rightarrow \Omega$ is now defined according to $P_{a}(x)=P_{a, v_{x}}(x)$. For an illustration, see Figure 3 .

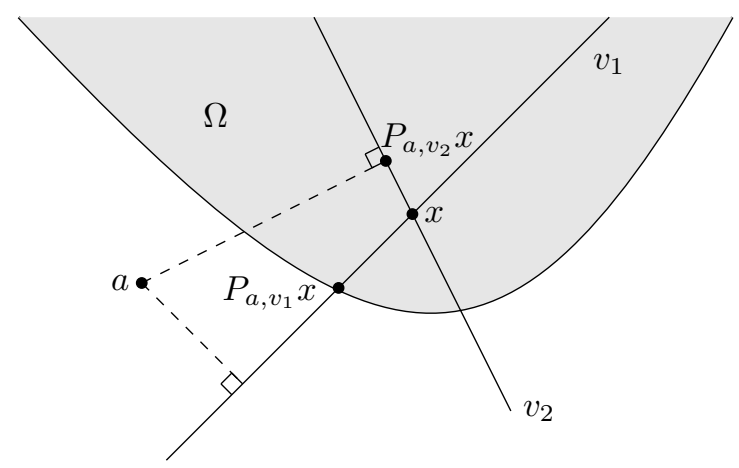

FigURE 3. Illustration of projections along special directions, in this case $P_{a}(x)=P_{a, v_{1}}(x)$.

We have now introduced all needed notation and proceed to the result:

Theorem 3.1. Let $\Omega \subset \mathbb{R}^{n}$ be closed and convex and satisfy the special cone property. Fix $a \in \mathbb{R}^{n}$. Choose any $x_{0} \in \Omega$. Let $x_{n}=P_{a}\left(x_{n-1}\right), n \in \mathbb{N}$. Then $x_{n} \rightarrow x_{*, a}$ as $n \rightarrow \infty$. 


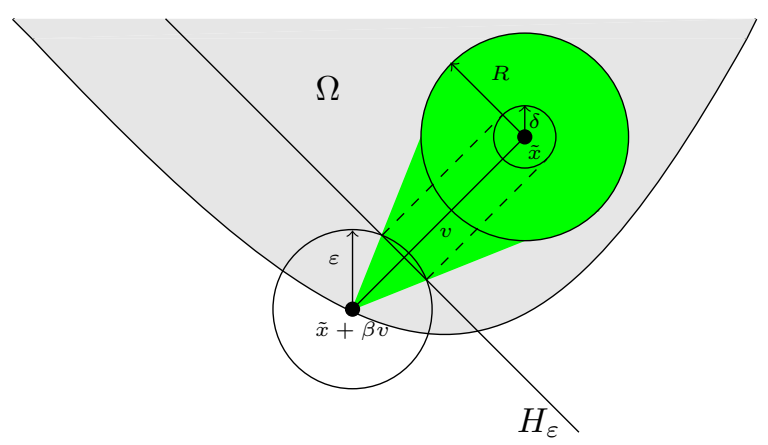

Figure 4. An illustration of the construction of the ball $B(\tilde{x}, \delta)$ with the set $\Lambda_{R}$ coloured in green.

Proof. The definition of $P_{a}$ implies

$$
\left\|x_{n}-a\right\|_{\ell^{2}}=\left\|P_{a}\left(x_{n-1}\right)-a\right\|_{\ell^{2}} \leq\left\|x_{n-1}-a\right\|_{\ell^{2}} .
$$

From (8) it follows that $\left\{x_{n}\right\}_{n \in \mathbb{N}}$ is bounded and since $\Omega$ is closed it therefore exists a subsequence $\left\{x_{n_{k}}\right\}_{k \in \mathbb{N}}$ such that $x_{n_{k}} \rightarrow \tilde{x} \in \Omega$ as $k \rightarrow \infty$. Further, in view of (8) we must have

$$
\|\tilde{x}-a\|_{\ell^{2}}=\inf _{n \in \mathbb{N}}\left\|x_{n}-a\right\|_{\ell^{2}} .
$$

Let us now show that $\tilde{x}=x_{*, a}$.

The first step is to show that $P_{a}(\tilde{x})=\tilde{x}$. Suppose the contrary, i.e. $P_{a}(\tilde{x}) \neq \tilde{x}$. Then there is a special direction $v$ such that $\tilde{x}+\beta v \in \Omega$ and $\|\tilde{x}+\beta v-a\|_{\ell^{2}}<$ $\|\tilde{x}-a\|_{\ell^{2}}$ for some small $\beta>0$. We now fix such $\beta$. Let $B(x, \varepsilon)$ denote the Euclidean ball centered at $x \in \mathbb{R}^{n}$ with radius $\varepsilon$. From continuity of $\|\cdot\|_{\ell^{2}}$ it follows that $\sup _{y \in B(\tilde{x}+\beta v, \varepsilon)}\|y-a\|_{\ell^{2}}=c\|\tilde{x}-a\|_{\ell^{2}}, c<1$, for $\varepsilon$ small enough. Given such $\varepsilon$, choosing $\delta>0$ small enough ensures

$$
\sup _{y \in B(\tilde{x}+\beta v, \varepsilon)}\|y-a\|_{\ell^{2}}=c\|\tilde{x}-a\|_{\ell^{2}}<\inf _{z \in B(\tilde{x}, \delta)}\|z-a\|_{\ell^{2}} .
$$

Further, convexity of $\Omega$ and choosing $\delta>0$ small enough give for any $z \in \Omega \cap B(\tilde{x}, \delta)$ that

$$
\inf _{u \in \Omega \cap\{z+t v: t \in \mathbb{R}\}}\|u-(\tilde{x}+\beta v)\|_{\ell^{2}}<\varepsilon,
$$

i.e. we can find an element $u$ on the line segment $\Omega \cap\{z+t v: t \in \mathbb{R}\}$ which is in the ball $B(\tilde{x}+\beta v, \varepsilon)$. This assertion is clear if $B(\tilde{x}+\beta v, \varepsilon) \subset \Omega$. If $B(\tilde{x}+\beta v, \varepsilon)$ is not contained in $\Omega$ some additional explanation is needed. Given fixed $R>0$, let $\Lambda_{R}$ denote the convex hull of the set $(\Omega \cap B(\tilde{x}, R)) \cup\{\tilde{x}+\beta v\}$. The convexity of $\Omega$ implies $\Lambda_{R} \subset \Omega$. Next, let $H_{\varepsilon}$ denote a hyperplane with normal direction $v$ which intersects the line segment $t \tilde{x}+(1-t)(\tilde{x}+\beta v), t \in(0,1)$, such that $\|\tilde{x}+\beta v-y\|_{\ell^{2}}<\varepsilon$ for every $y \in \Lambda_{R} \cap H_{\varepsilon}$. Choosing $\delta>0$ close enough to 0 ensures that the orthogonal projection of $\Omega \cap B(\tilde{x}, \delta)$ onto $H_{\varepsilon}$ will be contained in $\Lambda_{R}$. For such $\delta$, the condition (11) holds. An illustration of this construction is given in Figure 4. 
Take now an element $x \in\left\{x_{n_{k}}\right\}_{k \in \mathbb{N}} \cap B(\tilde{x}, \delta)$ where $\delta$ is chosen such that both (10) and (11) hold. Then we have

$$
\left\|P_{a}(x)-a\right\|_{\ell^{2}} \leq\left\|P_{a, v}(x)-a\right\|_{\ell^{2}} \leq c\|\tilde{x}-a\|_{\ell^{2}}<\|\tilde{x}-a\|_{\ell^{2}}
$$

which contradicts (9). So, $P_{a}(\tilde{x})=\tilde{x}$.

Consider now the set $S_{\tilde{x}}$ of all special directions $v$ for which $\tilde{x}+\beta v \in \Omega$ for small enough $\beta>0$. As the number of special directions is finite, we can fix the number $\beta$. We enumerate the special directions in $S_{\tilde{x}}$ according to $\left\{v_{1}, v_{2}, \ldots, v_{k}\right\}$. Our next step is to show that

$$
\|x-a\|_{\ell^{2}} \geq\|\tilde{x}-a\|_{\ell^{2}}
$$

for any $x=\tilde{x}+\sum_{i=1}^{k} \lambda_{i} v_{i}$ with $0 \leq \lambda_{i} \leq \beta, i=1, \ldots, k$.

Take an arbitrary $v \in S_{\tilde{x}}$. Note that from convexity of $\Omega$ it follows that $\tilde{x}+\lambda v \in \Omega$ for every $\lambda \in[0, \beta]$. Taking into account $P_{a}(\tilde{x})=\tilde{x}$, we have

and therefore

$$
\|\tilde{x}+\lambda v-a\|_{\ell^{2}} \geq\|\tilde{x}-a\|_{\ell^{2}}
$$

$$
\begin{aligned}
& \langle\tilde{x}+\lambda v-a, \tilde{x}+\lambda v-a\rangle= \\
& =\langle\tilde{x}-a, \tilde{x}-a\rangle+2 \lambda\langle\tilde{x}-a, v\rangle+\lambda^{2}\langle v, v\rangle \geq\langle\tilde{x}-a, \tilde{x}-a\rangle
\end{aligned}
$$

for every $\lambda \in[0, \beta]$. Since $\lambda$ can be arbitrary close to 0 it follows that

$$
\langle\tilde{x}-a, v\rangle \geq 0 \text {. }
$$

Consider next an arbitrary element $x$ of the form

$$
x=\tilde{x}+\sum_{i=1}^{k} \lambda_{i} v_{i}
$$

with $0 \leq \lambda_{i} \leq \beta, i=1, \ldots, k$. We have

$$
\begin{aligned}
& \|x-a\|_{\ell^{2}}^{2}=\left\|\tilde{x}+\sum_{i=1}^{k} \lambda_{i} v_{i}-a\right\|_{\ell^{2}}^{2}=\left\langle\tilde{x}+\sum_{i=1}^{k} \lambda_{i} v_{i}-a, \tilde{x}+\sum_{i=1}^{k} \lambda_{i} v_{i}-a\right\rangle= \\
& =\langle\tilde{x}-a, \tilde{x}-a\rangle+2 \sum_{i=1}^{k} \lambda_{i}\left\langle\tilde{x}-a, v_{i}\right\rangle+\sum_{i, j=1}^{k} \lambda_{i} \lambda_{j}\left\langle v_{i}, v_{j}\right\rangle .
\end{aligned}
$$

From (12) it follows that

$$
\sum_{i=1}^{k} \lambda_{i}\left\langle\tilde{x}-a, v_{i}\right\rangle \geq 0
$$

Further, $\sum_{i, j=1}^{k} \lambda_{i} \lambda_{j}\left\langle v_{i}, v_{j}\right\rangle$ is the quadratic form $\lambda^{T} M \lambda$ where $\lambda=\left(\lambda_{i}\right) \in \mathbb{R}^{k}$ and $M=\left(\left\langle v_{i}, v_{j}\right\rangle\right) \in \mathbb{R}^{k \times k}$. As $M$ is a Gram matrix and therefore positive semidefinite, see e.g. [10, Theorem 7.2.10], we have

$$
\sum_{i, j=1}^{k} \lambda_{i} \lambda_{j}\left\langle v_{i}, v_{j}\right\rangle \geq 0 .
$$

Taking into account (13) and (14) implies that

$$
\|x-a\|_{\ell^{2}} \geq\|\tilde{x}-a\|_{\ell^{2}}
$$

for any $x=\tilde{x}+\sum_{i=1}^{k} \lambda_{i} v_{i}$ with $0 \leq \lambda_{i} \leq \beta, i=1, \ldots, k$. 
Assume now that there exists $z \in \Omega$ such that

$$
\|z-a\|_{\ell^{2}}<\|\tilde{x}-a\|_{\ell^{2}} .
$$

As $\Omega$ has the special cone property it follows that $z \in \tilde{x}+K_{\tilde{x}}$. So,

$$
z=\tilde{x}+\sum_{i=1}^{k} \mu_{i} v_{i}
$$

for some $\mu_{i} \geq 0, i=1, \ldots, k$. Let $y_{\alpha}=\alpha z+(1-\alpha) \tilde{x}$ for $\alpha \in[0,1]$. It follows that

$$
y_{\alpha}=\tilde{x}+\sum_{i=1}^{k} \alpha \mu_{i} v_{i} .
$$

For any $\alpha \in(0,1]$ we have

$$
\begin{array}{r}
\left\|y_{\alpha}-a\right\|_{\ell^{2}}=\|\alpha z+(1-\alpha) \tilde{x}-a\|_{\ell^{2}} \leq \alpha\|z-a\|_{\ell^{2}}+(1-\alpha)\|\tilde{x}-a\|_{\ell^{2}}< \\
<\alpha\|\tilde{x}-a\|_{\ell^{2}}+(1-\alpha)\|\tilde{x}-a\|_{\ell^{2}}=\|\tilde{x}-a\|_{\ell^{2}} .
\end{array}
$$

By choosing $\alpha$ close enough to 0 the bounds $0 \leq \alpha \mu_{i} \leq \beta, i=1, \ldots, k$, hold. Hence, (16) contradicts (15). So, $\tilde{x}$ is an element of best approximation of $a$ in the whole set $\Omega$ with respect to the $\ell^{2}$-norm. The strict convexity of $\ell^{2}$-norm then gives $\tilde{x}=x_{*, a}$.

What remains is to establish convergence of the entire sequence $\left\{x_{n}\right\}_{n \in \mathbb{N}}$. Since the convergent subsequence $\left\{x_{n_{k}}\right\}_{k \in \mathbb{N}}$ was chosen arbitrary it follows that all convergent subsequences of $\left\{x_{n}\right\}_{n \in \mathbb{N}}$ converges to $x_{*, a}$. Suppose now that $x_{n} \nrightarrow x_{*, a}$. Then for $\eta>0$ small enough there exists a subsequence $\left\{x_{n_{l}}\right\}_{l \in \mathbb{N}}$ of $\left\{x_{n}\right\}_{n \in \mathbb{N}}$ such that

$$
\left\|x_{n_{l}}-x_{*, a}\right\|_{\ell^{2}} \geq \eta, \forall l \in \mathbb{N} .
$$

But $\left\{x_{n_{l}}\right\}_{l \in \mathbb{N}}$, as it is bounded, must have a subsequence $\left\{x_{n_{l_{m}}}\right\}_{m \in \mathbb{N}}$ with $x_{n_{l_{m}}} \rightarrow$ $x_{*, a}$ which contradicts (17). So, $x_{n} \rightarrow x_{*, a}$ as $n \rightarrow \infty$.

Remark 3.1. In applications, the algorithm can be simplified when taking into account the specific structure of the set $\Omega$. For an example, see [17] where a special version of the algorithm is applied to the Rudin-Osher-Fatemi image denoising model.

Considering the taut string problem with fixed ends, a different algorithm that computes the element $x_{*, a}$ in a finite number of steps can be given, see pp. 421-422 in 13 .

\section{Sufficient Conditions in the Continuous Setting}

We now turn to the study of invariant $K$-minimal sets with respect to the couple $\left(L^{1}\left([0,1]^{m}\right), L^{\infty}\left([0,1]^{m}\right)\right)$. Note that real $L^{p}$-spaces are considered in this paper. The present section is divided into three subsections. In Section 4.1 we introduce some notation and lemmas that will be needed in the sequel. Next, in Section 4.2 we present a sufficient condition for a set $\Omega \subset L^{1}\left([0,1]^{m}\right)+L^{\infty}\left([0,1]^{m}\right)=L^{1}\left([0,1]^{m}\right)$, $m \in \mathbb{N}$, to be invariant $K$-minimal with respect to the couple $\left(L^{1}\left([0,1]^{m}\right), L^{\infty}\left([0,1]^{m}\right)\right)$. Finally, in Section 4.3 we provide a sufficient condition for a set $\Omega \subset L^{1}\left([0,1]^{m}\right)$ to satisfy an infinite-dimensional analogue of invariant $\varphi$-minimality. 
4.1. Notation and lemmas. We first give a definition of a space of piecewise constant functions on $[0,1]^{m}$ that we will frequently use in the sequel.

Definition 4.1. Let $S_{n}\left([0,1]^{m}\right)$ denote the $n^{m}$-dimensional space of piecewise constant functions on $[0,1]^{m}$ given by

$$
S_{n}\left([0,1]^{m}\right)=\left\{g: g=\sum_{i_{1}, \ldots, i_{m}=1}^{n} c_{i_{1} \ldots i_{m}} \chi_{\Gamma_{i_{1} \ldots i_{m}}^{n}}, c_{i_{1} \ldots i_{m}} \in \mathbb{R}\right\}
$$

where $\Gamma_{i_{1} \ldots i_{m}}^{n}:=\left[\frac{i_{1}-1}{n}, \frac{i_{1}}{n}\right) \times \ldots \times\left[\frac{i_{m}-1}{n}, \frac{i_{m}}{n}\right), i_{1}, \ldots, i_{m}=1, \ldots, n$, and $\chi_{\Gamma_{i_{1} \ldots i_{m}}^{n}}$ is the characteristic function of $\Gamma_{i_{1} \ldots i_{m}}^{n}$.

For each $g=\sum_{i_{1}, \ldots, i_{m}=1}^{n} c_{i_{1} \ldots i_{m}} \chi_{\Gamma_{i_{1} \ldots i_{m}}^{n}} \in S_{n}\left([0,1]^{m}\right)$, we have a corresponding uniquely determined coefficient vector $c=\left(c_{1 \ldots 1}, \ldots, c_{n \ldots n}\right) \in \mathbb{R}^{n^{m}}$. This simple observation is the basis for the following definition:

Definition 4.2. Given $\Gamma \subset S_{n}\left([0,1]^{m}\right)$, we denote by $\widetilde{\Gamma}$ the set in $\mathbb{R}^{n^{m}}$ which consists of all coefficient vectors corresponding to the elements in $\Gamma$.

We can now formulate and prove the following lemma.

Lemma 4.1. Let $\Gamma \subset S_{n}\left([0,1]^{m}\right)$ be such that $\widetilde{\Gamma}$ is an invariant $K$-minimal set with respect to $\left(\ell^{1}, \ell^{\infty}\right)$ on $\mathbb{R}^{n^{m}}$. Then for any given $f \in S_{n}$, there exist $g_{*, f} \in \Gamma$ such that

$$
K\left(t, g_{*, f}-f ; L^{1}, L^{\infty}\right) \leq K\left(t, g-f ; L^{1}, L^{\infty}\right)
$$

for every $t>0$ and $g \in \Gamma$.

Proof. In the proof we will use the notation $\tilde{f}$ to denote the coefficient vector in $\mathbb{R}^{n^{m}}$ of $f \in S_{n}\left([0,1]^{m}\right)$.

Recall the well-known formula, see e.g. 2, Theorem 5.1.6, p. 298],

$$
K\left(t, h ; L^{1}, L^{\infty}\right)=\int_{0}^{t} h^{*}(s) d s
$$

where $h^{*}$ denotes the decreasing rearrangement of $h \in L^{1}+L^{\infty}$.

Let $h=\sum_{i=1}^{n^{m}} c_{i_{1} \ldots i_{m}} \chi_{\Gamma_{i_{1} \ldots i_{m}}^{n}} \in S_{n}$ be given. From (18) it follows that

$$
K\left(t, h ; L^{1}, L^{\infty}\right)=\left\{\begin{array}{l}
t c_{1}^{*}, 0<t \leq \frac{1}{n^{m}}, \\
\sum_{i=1}^{k} \frac{c_{i}^{*}}{n^{m}}+\left(t-\frac{k}{n^{m}}\right) c_{k+1}^{*}, \frac{k}{n^{m}}<t \leq \frac{k+1}{n^{m}}, \\
k=1, \ldots, n^{m}-1, \\
\sum_{i=1}^{n^{m}} \frac{c_{i}^{*}}{n^{m}}, t>1
\end{array}\right.
$$

where $c^{*} \in \mathbb{R}^{n^{m}}$ is the vector consisting of the coefficients $\left|c_{i_{1} \ldots . i_{m}}\right|, i_{1}, \ldots, i_{m}=$ $1, \ldots n$, sorted in decreasing order.

For the coefficient vector $\tilde{h} \in \mathbb{R}^{n^{m}}$ of $h$ we can show using (18) that (20)

$$
K\left(t, \tilde{h} ; \ell^{1}, \ell^{\infty}\right)=\left\{\begin{array}{l}
t c_{1}^{*}, 0<t \leq 1, \\
\sum_{i=1}^{k} c_{i}^{*}+(t-k) c_{k+1}^{*}, k<t \leq k+1, k=1, \ldots, n^{m}-1, \\
\sum_{i=1}^{n^{m}} c_{i}^{*}, t>n^{m} .
\end{array}\right.
$$

Suppose now $\Gamma \subset S_{n}$ is such that the corresponding set of coefficient vectors $\widetilde{\Gamma}$ is an invariant $K$-minimal set with respect to $\left(\ell^{1}, \ell^{\infty}\right)$ on $\mathbb{R}^{n^{m}}$. Take $f \in S_{n}$. Then 
there exists $\tilde{g}_{*, f} \in \widetilde{\Gamma}$ such that

$$
K\left(t, \tilde{g}_{*, f}-\tilde{f} ; \ell^{1}, \ell^{\infty}\right) \leq K\left(t, \tilde{g}-\tilde{f} ; \ell^{1}, \ell^{\infty}\right)
$$

for every $t>0$ and $\tilde{g} \in \widetilde{\Gamma}$. Comparing (19) with (20) it is then clear that

$$
K\left(t, g_{*, f}-f ; L^{1}, L^{\infty}\right) \leq K\left(t, g-f ; L^{1}, L^{\infty}\right)
$$

for every $t>0$ and $g \in \Gamma$.

Let $\mathcal{M}$ denote the set of real-valued Lebesgue measurable functions on $[0,1]^{m}$. We define the averaging operator $P_{n}: \mathcal{M} \rightarrow S_{n}$ by

$$
P_{n} f:=\sum_{i_{1}, \ldots, i_{m}=1}^{n} \frac{1}{\left(\frac{1}{n}\right)^{m}}\left(\int_{\Gamma_{i_{1} \ldots i_{m}}^{n}} f(s) d s\right) \chi_{\Gamma_{i_{1} \ldots i_{m}}^{n}} .
$$

The following result collects some properties of $P_{n}$ that we will need in the sequel:

Lemma 4.2. The operator $P_{n}$ satisfies $\left\|P_{n}\right\|_{L^{1} \rightarrow L^{1}} \leq 1,\left\|P_{n}\right\|_{L^{\infty} \rightarrow L^{\infty}} \leq 1$ and $P_{n} f \stackrel{L^{1}}{\rightarrow} f$.

Proof. The lemma follows easily from the definition of $P_{n}$ and direct computations.

\subsection{A sufficient condition for invariant $K$-minimal sets with respect to}

$\left(L^{1}, L^{\infty}\right)$. We are now ready to formulate and prove the following result:

Theorem 4.1. Let $\left\{\Omega_{2^{n}}\right\}_{n \in \mathbb{N}}$ be a family of sets such that for each $n \in \mathbb{N}$,

(i) $\Omega_{2^{n}}$ is a closed and convex subset of $S_{2^{n}}\left([0,1]^{m}\right)$ and the operator $P_{2^{n}}$ maps $\Omega_{2^{n+1}}$ into $\Omega_{2^{n}}$, i.e. $P_{2^{n}}: \Omega_{2^{n+1}} \rightarrow \Omega_{2^{n}}$,

(ii) the set of coefficient vectors $\widetilde{\Omega}_{2^{n}}$ is invariant $K$-minimal with respect to $\left(\ell^{1}, \ell^{\infty}\right)$ on $\mathbb{R}^{2^{n m}}$.

Then the set

$$
\Omega:=\left\{h \in L^{1}\left([0,1]^{m}\right): P_{2^{n}} h \in \Omega_{2^{n}}, \forall n \in \mathbb{N}\right\}
$$

is invariant $K$-minimal with respect to $\left(L^{1}\left([0,1]^{m}\right), L^{\infty}\left([0,1]^{m}\right)\right)$.

Proof. Let $f \in L^{1}\left([0,1]^{m}\right)$ be given. The assumption (ii) and Lemma 4.1 imply the existence of an element $g_{*, f}^{2^{n}} \in \Omega_{2^{n}}$ such that

$$
K\left(t, g_{*, f}^{2^{n}}-P_{2^{n}} f ; L^{1}, L^{\infty}\right) \leq K\left(t, g-P_{2^{n}} f ; L^{1}, L^{\infty}\right)
$$

for every $t>0$ and any $g \in \Omega_{2^{n}}$. We now show uniform boundedness in $L^{1}$ and uniform integrability of the sequence $\left\{g_{*, f}^{2^{n}}-P_{2^{n}} f\right\}_{n \in \mathbb{N}}$ which guarantees the existence of a weakly convergent subsequence $\left\{g_{*, f}^{2^{n_{k}}}-P_{2^{n_{k}}} f\right\}_{k \in \mathbb{N}}$ in $L^{1}$, see [8, Corollary IV.8.11, p. 294].

Consider first uniform boundedness in $L^{1}$. By recalling that for the decreasing rearrangement $F^{*}$ of $F \in L^{1}$ we have $\left\|F^{*}\right\|_{L^{1}[0, \infty)}=\|F\|_{L^{1}}$ and utilizing (18), (22) and $\left\|P_{2^{n}}\right\|_{L^{1} \rightarrow L^{1}} \leq 1$, we derive for any $h \in \Omega$ (clearly it is enough to consider the case $\Omega \neq \emptyset$ )

$$
\begin{array}{r}
\left\|g_{*, f}^{2^{n}}-P_{2^{n}} f\right\|_{L^{1}}=\left\|\left(g_{*, f}^{2^{n}}-P_{2^{n}} f\right)^{*}\right\|_{L^{1}[0, \infty)} \leq \\
\leq\left\|\left(P_{2^{n}} h-P_{2^{n}} f\right)^{*}\right\|_{L^{1}[0, \infty)}=\left\|P_{2^{n}} h-P_{2^{n}} f\right\|_{L^{1}} \leq\|h-f\|_{L^{1}} .
\end{array}
$$


So, $\left\{g_{*, f}^{2^{n}}-P_{2^{n}} f\right\}_{n \in \mathbb{N}}$ is uniformly bounded in $L^{1}$.

Next, consider uniform integrability. Let $A$ be any Lebesgue measurable subset of $[0,1]^{m}$ with measure $\mu(A)<t$ for $t>0$. From the Hardy-Littlewood rearrangement inequality, see e.g. [2, Theorem 2.2.2, p. 44], it follows for $\psi \in L^{1}\left([0,1]^{m}\right)$ that

$$
\int_{A}|\psi(x)| d x \leq \int_{0}^{t} \psi^{*}(s) d s
$$

Combining (23), (18) and (22) give

$$
\begin{aligned}
& \int_{A}\left|g_{*, f}^{2^{n}}(x)-P_{2^{n}} f(x)\right| d x \leq \int_{0}^{t}\left(g_{*, f}^{2^{n}}-P_{2^{n}} f\right)^{*}(s) d s= \\
= & K\left(t, g_{*, f}^{2^{n}}-P_{2^{n}} f ; L^{1}, L^{\infty}\right) \leq K\left(t, P_{2^{n}} h-P_{2^{n}} f ; L^{1}, L^{\infty}\right)
\end{aligned}
$$

for any $h \in \Omega$. As $\max \left\{\left\|P_{2^{n}}\right\|_{L^{1} \rightarrow L^{1}},\left\|P_{2^{n}}\right\|_{L^{\infty} \rightarrow L^{\infty}}\right\} \leq 1$ we have

$$
\begin{array}{r}
K\left(t, P_{2^{n}} h-P_{2^{n}} f ; L^{1}, L^{\infty}\right) \leq \inf _{h-f=g_{0}+g_{1}}\left(\left\|P_{2^{n}} g_{0}\right\|_{L^{1}}+t\left\|P_{2^{n}} g_{1}\right\|_{L^{\infty}}\right) \leq \\
\leq \inf _{h-f=g_{0}+g_{1}}\left(\left\|g_{0}\right\|_{L^{1}}+t\left\|g_{1}\right\|_{L^{\infty}}\right)=K\left(t, h-f ; L^{1}, L^{\infty}\right)
\end{array}
$$

which combined with (24) and (18) give

$$
\int_{A}\left|g_{*, f}^{2^{n}}(x)-P_{2^{n}} f(x)\right| d x \leq K\left(t, h-f ; L^{1}, L^{\infty}\right)=\int_{0}^{t}(h-f)^{*}(s) d s .
$$

Together, (26) and

$$
\lim _{t \rightarrow 0^{+}} \int_{0}^{t}(h-f)^{*}(s) d s=0
$$

imply that for any $\varepsilon>0$ it is possible to find $\delta=\delta(\varepsilon)>0$ such that if $\mu(A)<\delta$ then

$$
\int_{A}\left|g_{*, f}^{2^{n}}(x)-P_{2^{n}} f(x)\right| d x \leq \varepsilon
$$

for all $n \in \mathbb{N}$. Hence, $\left\{g_{*, f}^{2^{n}}-P_{2^{n}} f\right\}_{n \in \mathbb{N}}$ is uniformly integrable. So, there exists a subsequence $\left\{g_{*, f}^{2^{n_{k}}}-P_{2^{n_{k}}} f\right\}_{k \in \mathbb{N}}$ that converges weakly in $L^{1}$, i.e.

$$
h^{2^{n_{k}}}:=g_{*, f}^{2^{n_{k}}}-P_{2^{n_{k}}} f \stackrel{L^{1}}{\rightarrow} \bar{h} \in L^{1}\left([0,1]^{m}\right) .
$$

Recalling that $P_{n} f \stackrel{L^{1}}{\rightarrow} f$, cf. Lemma 4.2 , gives

$$
g_{*, f}^{2^{n_{k}}}=h^{2^{n_{k}}}+P_{2^{n_{k}}} f \stackrel{L^{1}}{\rightarrow} \bar{h}+f:=g_{*, f} \in L^{1}\left([0,1]^{m}\right) .
$$

We now show that $g_{*, f} \in \Omega$. Mazur's lemma, see [8, Corollary V.3.14, p. 422], implies that

$$
\sum_{l=k}^{N(k)} \lambda_{l}(k) g_{*, f}^{2^{n_{l}}} \stackrel{L^{1}}{\rightarrow} g_{*, f}
$$

where $N: \mathbb{N} \rightarrow \mathbb{N}$, with $N(n) \geq n$, and the sequence of sets of real numbers $\left\{\lambda_{l}(k): l=k, \ldots, N(k)\right\}_{k \in \mathbb{N}}$ satisfy $\lambda_{l}(k) \geq 0$ and $\sum_{l=k}^{N(k)} \lambda_{l}(k)=1$. That is, the sequence of convex combinations $\left\{\sum_{l=k}^{N(k)} \lambda_{l}(k) g_{*, f}^{2^{n_{l}}}\right\}_{k \in \mathbb{N}}$ converges strongly to $g_{*, f}$ in $L^{1}$. 
Let now $M \in \mathbb{N}$ be given. With (27) at our disposal, we can for any $\varepsilon>0$ find $k_{M}=k_{M}(\varepsilon) \in \mathbb{N}$ with $n_{k_{M}} \geq M$ such that

$$
\left\|\sum_{l=k_{M}}^{N\left(k_{M}\right)} \lambda_{l}\left(k_{M}\right) g_{*, f}^{2^{n_{l}}}-g_{*, f}\right\|_{L^{1}} \leq \varepsilon
$$

The identity $P_{2^{n}}\left(P_{2^{n+1}}\right)=P_{2^{n}}$ (recall the definition of $P_{n}$ in (21) $)$ and the assumption $P_{2^{n}}: \Omega_{2^{n+1}} \rightarrow \Omega_{2^{n}}$ for each $n \in \mathbb{N}$ give, taking into account $n_{k_{M}} \geq M$, that $P_{2^{M}} g_{*, f}^{2^{2_{l}}} \in \Omega_{2^{M}}, l=k_{M}, \ldots, N\left(k_{M}\right)$. This gives in turn

$$
P_{2^{M}}\left(\sum_{l=k_{M}}^{N\left(k_{M}\right)} \lambda_{l}\left(k_{M}\right) g_{*, f}^{2^{n_{l}}}\right)=\sum_{l=k_{M}}^{N\left(k_{M}\right)} \lambda_{l}\left(k_{M}\right) P_{2^{M}} g_{*, f}^{2^{n_{l}}} \in \Omega_{2^{M}}
$$

because $\Omega_{2^{n}}$ by assumption is a convex subset of $S_{2^{n}}$ for each $n \in \mathbb{N}$. Moreover, $\left\|P_{n}\right\|_{L^{1} \rightarrow L^{1}} \leq 1$ gives

$$
\left\|P_{2^{M}}\left(\sum_{l=k_{M}}^{N\left(k_{M}\right)} \lambda_{l}\left(k_{M}\right) g_{*, f}^{2^{n_{l}}}\right)-P_{2^{M}} g_{*, f}\right\|_{L^{1}} \leq\left\|\sum_{l=k_{M}}^{N\left(k_{M}\right)} \lambda_{l}\left(k_{M}\right) g_{*, f}^{2^{n_{l}}}-g_{*, f}\right\|_{L^{1}} \leq \varepsilon .
$$

Since $\varepsilon$ can be chosen arbitrary close to 0 and taking into account (28) and (29) we obtain

$$
\inf _{g \in \Omega_{2^{M}}}\left\|g-P_{2^{M}} g_{*, f}\right\|_{L^{1}}=0 .
$$

Hence, $P_{2^{M}} g_{*, f} \in \Omega_{2^{M}}$ as $\Omega_{2^{n}}$ by assumption is a closed subset of $S_{2^{n}}$ for each $n \in \mathbb{N}$. The choice of $M \in \mathbb{N}$ was arbitrary and we conclude therefore that

$$
g_{*, f} \in \Omega=\left\{h \in L^{1}\left([0,1]^{m}\right): P_{2^{n}} h \in \Omega_{2^{n}}, \forall n \in \mathbb{N}\right\} .
$$

Let us now show that $g_{*, f}-f$ is an element with minimal $K$-functional in the set $\Omega-f$. Recall that $K\left(t, \cdot ; L^{1}, L^{\infty}\right)$ for every fixed $t>0$ is an equivalent norm of $L^{1}$ on $L^{1}\left([0,1]^{m}\right)+L^{\infty}\left([0,1]^{m}\right)=L^{1}\left([0,1]^{m}\right)$. Combining (27) with

$$
\sum_{l=k}^{N(k)} \lambda_{l}(k) P_{2^{n_{l}}} f \stackrel{L^{1}}{\rightarrow} f
$$

which follows from $P_{n} f \stackrel{L^{1}}{\rightarrow} f$, give

$$
K\left(t, g_{*, f}-f ; L^{1}, L^{\infty}\right)=\lim _{k \rightarrow \infty} K\left(t, \sum_{l=k}^{N(k)} \lambda_{l}(k)\left(g_{*, f}^{2^{n_{l}}}-P_{2^{n_{l}}} f\right) ; L^{1}, L^{\infty}\right)
$$

for every $t>0$. Further, applying the triangle inequality, (22) and (25) in turn we obtain

$$
\begin{aligned}
& K\left(t, \sum_{l=k}^{N(k)} \lambda_{l}(k)\left(g_{*, f}^{2^{n_{l}}}-P_{2^{n_{l}}} f\right) ; L^{1}, L^{\infty}\right) \leq \sum_{l=k}^{N(k)} \lambda_{l}(k) K\left(t, g_{*, f}^{2^{n_{l}}}-P_{2^{n_{l}}} f ; L^{1}, L^{\infty}\right) \leq \\
& \leq \sum_{l=k}^{N(k)} \lambda_{l}(k) K\left(t, P_{2^{n_{l}}} h-P_{2^{n_{l}}} f ; L^{1}, L^{\infty}\right) \leq \sum_{l=k}^{N(k)} \lambda_{l}(k) K\left(t, h-f ; L^{1}, L^{\infty}\right)= \\
& =K\left(t, h-f ; L^{1}, L^{\infty}\right)
\end{aligned}
$$


for every $h \in \Omega$ and $t>0$. So, taking into account (30) we obtain

$$
K\left(t, g_{*, f}-f ; L^{1}, L^{\infty}\right) \leq K\left(t, h-f ; L^{1}, L^{\infty}\right)
$$

for every $h \in \Omega$ and $t>0$. Hence, $g_{*, f}$ is an element with minimal $K$-minimal element in the set $\Omega-f$.

As $f \in L^{1}\left([0,1]^{m}\right)$ was chosen arbitrarily we conclude that $\Omega$ is an invariant $K$-minimal set with respect to $\left(L^{1}\left([0,1]^{m}\right), L^{\infty}\left([0,1]^{m}\right)\right)$.

4.3. A sufficient condition for invariant $\varphi$-minimal sets in the continuous setting. We now introduce invariant $\varphi$-minimal sets in the setting of $L^{1}\left([0,1]^{m}\right)$, compare with Definition 2.1.

Definition 4.3. A set $\Omega \subset L^{1}\left([0,1]^{m}\right)$ is called invariant $\varphi$-minimal if for every element $f \in L^{1}\left([0,1]^{m}\right)$, there exists an element $g_{f}^{*} \in \Omega$ such that

$$
\int_{[0,1]^{m}} \varphi\left(g_{f}^{*}(x)-f(x)\right) d x \leq \int_{[0,1]^{m}} \varphi(g(x)-f(x)) d x
$$

for every $g \in \Omega$ and every convex function $\varphi: \mathbb{R} \rightarrow \mathbb{R}$.

The proof of Theorem 4.2 below shares similarities with the proof of Theorem 4.1. However, since the functional $\int \varphi(\cdot)$ is not a norm on $L^{1}$ we need to use additional tools here. More precisely, we will also use Jensen's inequality and another inequality for convex functions originally formulated by Hardy, Littlewood and Pólya, see [9].

Theorem 4.2. Let $\left\{\Omega_{2^{n}}\right\}_{n \in \mathbb{N}}$ be a family of sets such that for each $n \in \mathbb{N}$,

(i) $\Omega_{2^{n}}$ is a closed and convex subset of $S_{2^{n}}\left([0,1]^{m}\right)$ and $P_{2^{n}}: \Omega_{2^{n+1}} \rightarrow \Omega_{2^{n}}$,

(ii) $\widetilde{\Omega}_{2^{n}}$ has the special cone property,

(iii) $\int_{[0,1]^{m}} g(x) d x=C$ for every $g \in \Omega_{2^{n}}$.

Then the set

is invariant $\varphi$-minimal.

$$
\Omega:=\left\{h \in L^{1}\left([0,1]^{m}\right): P_{2^{n}} h \in \Omega_{2^{n}}, \forall n \in \mathbb{N}\right\}
$$

Proof. Let $f \in L^{1}\left([0,1]^{m}\right)$ be given. Conditions (ii) and (iii) give that $\widetilde{\Omega}_{2^{n}}$ is invariant $\varphi$-minimal on $\mathbb{R}^{2 m}$ by Theorem 2.1. In particular is $\widetilde{\Omega}_{2^{n}}$ invariant $K$ minimal with respect to $\left(\ell^{1}, \ell^{\infty}\right)$ on $\mathbb{R}^{2^{n m}}$. So, by Lemma 4.1 there exists an element $g_{*, f}^{2^{n}} \in \Omega_{2^{n}}$ such that

$$
K\left(t, g_{*, f}^{2^{n}}-P_{2^{n}} f ; L^{1}, L^{\infty}\right) \leq K\left(t, g-P_{2^{n}} f ; L^{1}, L^{\infty}\right)
$$

for every $g \in \Omega_{2^{n}}$ and $t>0$. As $\widetilde{\Omega}_{2^{n}}$ is invariant $\varphi$-minimal on $\mathbb{R}^{2^{n m}}$ it is possible to show, by arguments similar to the proof of Lemma 4.1 that the element $g_{*, f}^{2^{n}}$ in addition satisfy

$$
\int_{[0,1]^{m}} \varphi\left(g_{*, f}^{2^{n}}(x)-P_{2^{n}} f(x)\right) d x \leq \int_{[0,1]^{m}} \varphi\left(g(x)-P_{2^{n}} f(x)\right) d x
$$

for every $g \in \Omega_{2^{n}}$ and every convex function $\varphi: \mathbb{R} \rightarrow \mathbb{R}$.

With the same arguments as in the proof of Theorem 4.1, i.e. uniform boundedness in $L^{1}$ and uniform integrability, one can show the existence of a subsequence $\left\{g_{*}^{2^{n_{k}}}\right\}_{k \in \mathbb{N}}$ such that

$$
g_{*, f}^{2^{n_{k}}} \stackrel{L^{1}}{\rightarrow} g_{*, f} \in \Omega
$$


Mazur's lemma then implies the existence of a function $N: \mathbb{N} \rightarrow \mathbb{N}, N(n) \geq n$, and a sequence of sets of real numbers $\left\{\lambda_{l}(k): l=k, \ldots, N(k)\right\}_{j \in \mathbb{N}}$, with $\lambda_{l}(k) \geq 0$ and $\sum_{l=k}^{N(k)} \lambda_{l}(k)=1$, such that the sequence $\left\{\sum_{l=k}^{N(k)} \lambda_{l}(k) g_{*, f}^{2^{n_{l}}}\right\}_{k \in \mathbb{N}}$ converges strongly to $g_{*, f}$ in $L^{1}$.

Using Jensen's inequality, $P_{2^{n}}: \Omega \rightarrow \Omega_{2^{n}}$ and (31) we derive

$$
\begin{array}{r}
\int_{[0,1]^{m}} \varphi\left(\sum_{l=k}^{N(k)} \lambda_{l}(k)\left(g_{*, f}^{2^{n_{l}}}(x)-P_{2^{n_{l}}} f(x)\right)\right) d x \leq \\
\leq \sum_{l=k}^{N(k)} \lambda_{l}(k) \int_{[0,1]^{m}} \varphi\left(g_{*, f}^{2^{n_{l}}}(x)-P_{2^{n_{l}}} f(x)\right) d x \leq \\
\leq \sum_{l=k}^{N(k)} \lambda_{l}(k) \int_{[0,1]^{m}} \varphi\left(P_{2^{n_{l}}} h(x)-P_{2^{n_{l}}} f(x)\right) d x
\end{array}
$$

for every $h \in \Omega$ and every convex function $\varphi: \mathbb{R} \rightarrow \mathbb{R}$. Further, the definition of $P_{2^{n}}$ and Jensen's inequality (for integrals) imply

$$
\begin{aligned}
& \int_{[0,1]^{m}} \varphi\left(P_{2^{n_{l}}} h(x)-P_{2^{n_{l}}} f(x)\right) d x= \\
& =\sum_{i_{1}, \ldots, i_{m}=1}^{2^{n_{l}}} \varphi\left(\frac{1}{\frac{1}{2^{n m}}} \int_{\Gamma_{i_{1} \ldots i_{m}}^{2^{n_{l}}}}(h(s)-f(s)) d s\right) \frac{1}{2^{n m}} \leq \\
& \leq \sum_{i_{1}, \ldots, i_{m}=1}^{2^{n_{l}}} \frac{1}{\frac{1}{2^{n m}}}\left(\int_{\Gamma_{i_{1} \ldots i_{m}}^{2^{n_{l}}}} \varphi(h(s)-f(s)) d s\right) \frac{1}{2^{n m}}=\int_{[0,1]^{m}} \varphi(h(s)-f(s)) d s
\end{aligned}
$$

for every $h \in \Omega$ and every convex function $\varphi: \mathbb{R} \rightarrow \mathbb{R}$. Combining (33) and (34) give for every $h \in \Omega$ and every convex function $\varphi: \mathbb{R} \rightarrow \mathbb{R}$

$$
\int_{[0,1]^{m}} \varphi\left(\sum_{l=k}^{N(k)} \lambda_{l}(k)\left(g_{*, f}^{2^{n_{l}}}(x)-P_{2^{n_{l}}} f(x)\right)\right) d x \leq \int_{[0,1]^{m}} \varphi(h(s)-f(s)) d s .
$$

Let us now consider the limiting behaviour of (35) when $k \rightarrow \infty$. In order to do so we recall a result first formulated in 9 . Given $f_{1}, f_{2} \in L^{1}[a, b]$, the inequality

$$
\int_{a}^{b} \varphi\left(f_{1}(x)\right) d x \leq \int_{a}^{b} \varphi\left(f_{2}(x)\right) d x
$$

holds for every convex function $\varphi: \mathbb{R} \rightarrow \mathbb{R}$ if and only if

$$
\int_{a}^{b} f_{1}(x) d x=\int_{a}^{b} f_{2}(x) d x
$$

and

$$
\int_{a}^{b} \max \left\{f_{1}(x)-u, 0\right\} d x \leq \int_{a}^{b} \max \left\{f_{2}(x)-u, 0\right\} d x
$$

for every $u \in \mathbb{R}$. Later on, it was found that the proof in 9] was done for a certain class of convex functions. For a complete proof, i.e. for all convex functions $\varphi: \mathbb{R} \rightarrow \mathbb{R}$, and generalization to finite measure spaces we refer to [14 and 6 . 
As (35) holds for every convex function $\varphi: \mathbb{R} \rightarrow \mathbb{R}$, it follows from the above cited characterization that

$$
\begin{array}{r}
\int_{[0,1]^{m}} \max \left\{\sum_{l=k}^{N(k)} \lambda_{l}(k)\left(g_{*, f}^{2^{l}}(x)-P_{2^{l}} f(x)\right)-u, 0\right\} d x \leq \\
\leq \int_{[0,1]^{m}} \max \{h(x)-f(x)-u, 0\} d x
\end{array}
$$

for any $u \in \mathbb{R}$ and any $h \in \Omega$. As $f_{n} \stackrel{L^{1}}{\rightarrow} f$ implies $\max \left\{f_{n}-u, 0\right\} \stackrel{L^{1}}{\rightarrow} \max \{f-u, 0\}$ for any given $u \in \mathbb{R}$, we have

$$
\max \left\{\sum_{l=k}^{N(k)} \lambda_{l}(k)\left(g_{*, f}^{2^{l}}-P_{2^{l}} f\right)-u, 0\right\} \stackrel{L^{1}}{\rightarrow} \max \left\{g_{*, f}-f-u, 0\right\} .
$$

for every $u \in \mathbb{R}$. Combining (36) and (37) then give

$$
\int_{[0,1]^{m}} \max \left\{g_{*, f}(x)-f(x)-u, 0\right\} d x \leq \int_{[0,1]^{m}} \max \{h(x)-f(x)-u, 0\} d x
$$

for every $u \in \mathbb{R}$ and any $h \in \Omega$. Further, as also $g_{*, f} \in \Omega$, recall (32), we have

$$
\int_{[0,1]^{m}} g_{*, f}(x) d x=\int_{[0,1]^{m}} P_{2^{n}} g_{*, f}(x) d x=C=\int_{[0,1]^{m}} P_{2^{n}} h(x) d x=\int_{[0,1]^{m}} h(x) d x
$$

Hence,

$$
\int_{[0,1]^{m}}\left(g_{*, f}(x)-f(x)\right) d x=\int_{[0,1]^{m}}(h(x)-f(x)) d x .
$$

From (38) and (39) we then conclude, utilizing the above cited characterization, that

$$
\int_{[0,1]^{m}} \varphi\left(g_{*, f}(x)-f(x)\right) d x \leq \int_{[0,1]^{m}} \varphi(h(x)-f(x)) d x
$$

for every convex function $\varphi: \mathbb{R} \rightarrow \mathbb{R}$ and every $h \in \Omega$. As $f \in L^{1}\left([0,1]^{m}\right)$ was chosen arbitrary the theorem now follows.

\section{ExAmples In The CONTINUOUS SETting}

We now construct examples of invariant $K$-minimal sets with respect to the couple $\left(L^{1}\left([0,1]^{m}\right), L^{\infty}\left([0,1]^{m}\right)\right)$ by using Theorem 4.1 These examples are infinitedimensional analogues of the finite-dimensional examples considered in Section 2 .

5.1. Rectangular domains in the continuous setting. We here provide an infinite-dimensional analogue of Proposition 2.1.

Proposition 5.1. Let $F, G \in L^{1}\left([0,1]^{m}\right)$ with $F \leq G$ almost everywhere (a.e.) on $[0,1]^{m}$. Then the set

$$
\Lambda:=\left\{g \in L^{1}\left([0,1]^{m}\right): F \leq g \leq G \text { a.e. on }[0,1]^{m}\right\}
$$

is invariant K-minimal with respect to $\left(L^{1}\left([0,1]^{m}\right), L^{\infty}\left([0,1]^{m}\right)\right)$. 
Proof. In order to make formulas clear we will consider the case $m=1$. The case $m>1$ is proved completely analogously.

Consider the family of sets $\left\{\Omega_{2^{n}}\right\}_{n \in \mathbb{N}}$ where

$$
\Omega_{2^{n}}:=\left\{h \in S_{2^{n}}[0,1]: P_{2^{n}} F \leq h \leq P_{2^{n}} G\right\} .
$$

It is clear from the definition that $\Omega_{2^{n}}$ is a closed and convex subset of $S_{2^{n}}$. Take $h \in \Omega_{2^{n+1}}$. Through direct computations one verifies that $P_{2^{n}} h \in \Omega_{2^{n}}$. Hence, $P_{2^{n}}: \Omega_{2^{n+1}} \rightarrow \Omega_{2^{n}}$. The coefficient set $\widetilde{\Omega}_{2^{n}}$ of $\Omega_{2^{n}}$ is given by

$$
\widetilde{\Omega}_{2^{n}}=\left\{c \in \mathbb{R}^{2^{n}}: \frac{1}{\frac{1}{2^{n}}} \int_{\Gamma_{i}^{2^{n}}} F(s) d s \leq c_{i} \leq \frac{1}{\frac{1}{2^{n}}} \int_{\Gamma_{i}^{2^{n}}} G(s) d s, i=1, \ldots, 2^{n}\right\} .
$$

Recall that $\Gamma_{i}^{2^{n}}=\left[\frac{i-1}{2^{n}}, \frac{i}{2^{n}}\right), i=1, \ldots, 2^{n}$. We notice that $\widetilde{\Omega}_{2^{n}}$ falls into the category of sets of Proposition 2.1. So $\widetilde{\Omega}_{2^{n}}$ is invariant $K$-minimal with respect to $\left(\ell^{1}, \ell^{\infty}\right)$ on $\mathbb{R}^{2^{n}}$.

From Theorem 4.1 it now follows that the set

$$
\Omega:=\left\{g \in L^{1}[0,1]: P_{2^{n}} g \in \Omega_{2^{n}}, \forall n \in \mathbb{N}\right\}
$$

is invariant $K$-minimal with respect to $\left(L^{1}, L^{\infty}\right)$. The remaining part of the proof is devoted to show the characterization $\Omega=\Lambda$.

First consider the inclusion $\Lambda \subset \Omega$. Take $g \in \Lambda$ and $n \in \mathbb{N}$. For $x \in \Gamma_{i}^{2^{n}}$ we have

$$
\begin{array}{r}
P_{2^{n}} F(x)=\frac{1}{\frac{1}{2^{n}}} \int_{\Gamma_{i}^{2^{n}}} F(s) d s \leq P_{2^{n}} g(x)=\frac{1}{\frac{1}{2^{n}}} \int_{\Gamma_{i}^{2^{n}}} g(s) d s \leq \frac{1}{\frac{1}{2^{n}}} \int_{\Gamma_{i}^{2^{n}}} G(s) d s= \\
=P_{2^{n}} G(x) .
\end{array}
$$

So, $P_{2^{n}} F \leq P_{2^{n}} g \leq P_{2^{n}} G$ and therefore $P_{2^{n}} g \in \Omega_{2^{n}}$. As $g \in \Lambda$ and $n \in \mathbb{N}$ were arbitrarily chosen we conclude that $\Lambda \subset \Omega$.

We now turn to the inclusion $\Lambda \supset \Omega$. Let $g \in \Omega$. Given $f \in L^{1}[0,1]$, $P_{2^{n}} f \stackrel{L^{1}}{\rightarrow} f$ implies, see [8, Corollary III.6.13, p. 150], the existence of a subsequence $\left\{P_{2^{n_{k}}} f\right\}_{k \in \mathbb{N}}$ such that

$$
f(x)=\lim _{k \rightarrow \infty} P_{2^{n_{k}}} f(x)
$$

almost everywhere on [0,1]. Combining (40) with $P_{2^{n}} F \leq P_{2^{n}} g \leq P_{2^{n}} G$ for every $n \in \mathbb{N}$, which follows from $g \in \Omega$, give

$$
F \leq g \leq G
$$

almost everywhere on $[0,1]$. We conclude that $g \in \Lambda$ and since $g \in \Omega$ was arbitrary we have $\Lambda \supset \Omega$.

We have now established $\Omega=\Lambda$ and the proposition follows.

Corollary 5.1. The $L^{\infty}$-ball with radius $t>0$ is an invariant $K$-minimal set with respect to $\left(L^{1}\left([0,1]^{m}\right), L^{\infty}\left([0,1]^{m}\right)\right)$.

Proof. The corollary follows from Proposition 5.1 by taking $F=-t$ and $G=t$. 
5.2. Intersection of rectangular domain and hyperplane in the continuous setting. Our second example concerns an infinite-dimensional analogue of Theorem 2.3. Compared with the finite-dimensional setting, we interchange the order between the corresponding analogues of Theorem 2.2 and Theorem 2.3 . We do so because the provided proof of the analogue of Theorem 2.3 is easier in our opinion.

Theorem 5.1. Let $F, G \in L^{1}\left([0,1]^{m}\right)$ with $F \leq G$ almost everywhere (a.e.) on $[0,1]^{m}$. Then the set

$$
\Lambda:=\left\{g \in L^{1}\left([0,1]^{m}\right): F \leq g \leq G \text { a.e. on }[0,1]^{m}, \int_{[0,1]^{m}} g(x) d x=C\right\},
$$

where the constant $C \in \mathbb{R}$ is such that $\int_{[0,1]^{m}} F(x) d x \leq C \leq \int_{[0,1]^{m}} G(x) d x$, is invariant $K$-minimal with respect to $\left(L^{1}\left([0,1]^{m}\right), L^{\infty}\left([0,1]^{m}\right)\right)$.

Proof. The proof will resemble the proof of the preceding proposition. As there, we will consider the case $m=1$ in order to make formulas clear. The case $m>1$ is proved completely analogously.

Consider the family of sets $\left\{\Omega_{2^{n}}\right\}_{n \in \mathbb{N}}$ where

$$
\Omega_{2^{n}}:=\left\{h \in S_{2^{n}}[0,1]: P_{2^{n}} F \leq h \leq P_{2^{n}} G, \quad \int_{0}^{1} h(x) d x=C\right\} .
$$

It is clear from the definition that $\Omega_{2^{n}}$ is a closed and convex subset of $S_{2^{n}}$. Take $h \in \Omega_{2^{n+1}}$. Through direct computations one verifies that $P_{2^{n}} h \in \Omega_{2^{n}}$. Hence, $P_{2^{n}}: \Omega_{2^{n+1}} \rightarrow \Omega_{2^{n}}$. The coefficient set $\widetilde{\Omega}_{2^{n}}$ of $\Omega_{2^{n}}$ is given by

$$
\begin{aligned}
& \widetilde{\Omega}_{2^{n}}=\left\{c \in \mathbb{R}^{2^{n}}: \frac{1}{\frac{1}{2^{n}}} \int_{\Gamma_{i}^{2^{n}}} F(s) d s \leq c_{i} \leq \frac{1}{\frac{1}{2^{n}}} \int_{\Gamma_{i}^{2^{n}}} G(s) d s, i=1, \ldots, 2^{n},\right. \\
& \left.\sum_{i=1}^{2^{n}} \frac{c_{i}}{2^{n}}=C\right\} .
\end{aligned}
$$

We notice that $\widetilde{\Omega}_{2^{n}}$ falls into the category of sets of Theorem 2.3 . So $\widetilde{\Omega}_{2^{n}}$ is invariant $K$-minimal with respect to $\left(\ell^{1}, \ell^{\infty}\right)$ on $\mathbb{R}^{2^{n}}$.

From Theorem 4.1 it now follows that the set

$$
\Omega:=\left\{g \in L^{1}[0,1]: P_{2^{n}} g \in \Omega_{2^{n}}, \forall n \in \mathbb{N}\right\}
$$

is invariant $K$-minimal with respect to $\left(L^{1}, L^{\infty}\right)$. The remaining part of the proof is devoted to show the characterization $\Omega=\Lambda$.

We first consider the inclusion $\Lambda \subset \Omega$. Take $g \in \Lambda$ and $n \in \mathbb{N}$. Then for $x \in \Gamma_{i}^{2^{n}}$ we have

$$
\frac{1}{\frac{1}{2^{n}}} \int_{\Gamma_{i}^{2^{n}}} F(s) d s \leq P_{2^{n}} g(x)=\frac{1}{\frac{1}{2^{n}}} \int_{\Gamma_{i}^{2^{n}}} g(s) d s \leq \frac{1}{\frac{1}{2^{n}}} \int_{\Gamma_{i}^{2^{n}}} G(s) d s .
$$

So, $P_{2^{n}} F \leq P_{2^{n}} g \leq P_{2^{n}} G$. Moreover,

$$
\int_{0}^{1} P_{2^{n}} g(x) d x=\int_{0}^{1} g(x) d x=C .
$$

Hence, $P_{2^{n}} g \in \Omega_{2^{n}}$. As $g \in \Lambda$ and $n \in \mathbb{N}$ were arbitrarily chosen we conclude that $\Lambda \subset \Omega$. 
We now turn to the inclusion $\Lambda \supset \Omega$. Let $g \in \Omega$. From $P_{2^{n}} g \stackrel{L^{1}}{\rightarrow} g$ it follows that

$$
\int_{0}^{1} g(x) d x=\lim _{n \rightarrow \infty} \int_{0}^{1} P_{2^{n}} g(x) d x=C .
$$

Further, given $f \in L^{1}[0,1], P_{2^{n}} f \stackrel{L^{1}}{\rightarrow} f$ implies, see [8, Corollary III.6.13, p. 150], the existence of a subsequence $\left\{P_{2^{n}{ }_{k}} f\right\}_{k \in \mathbb{N}}$ such that

$$
f(x)=\lim _{k \rightarrow \infty} P_{2^{n_{k}}} f(x)
$$

almost everywhere on $[0,1]$. Combining (43) with $P_{2^{n}} F \leq P_{2^{n}} g \leq P_{2^{n}} G$ for every $n \in \mathbb{N}$, which follows from $g \in \Omega$, give

$$
F \leq g \leq G
$$

almost everywhere on $[0,1]$. Taking into account (42) and (44) we conclude that $g \in \Lambda$. Since $g \in \Omega$ was arbitrary we have $\Lambda \supset \Omega$.

We have now established $\Omega=\Lambda$ and the theorem follows.

Remark 5.1. From the proof of Theorem 5.1 we know that the family of sets $\left\{\Omega_{2^{n}}\right\}_{n \in \mathbb{N}}$ defined by (41) satisfy the conditions of Theorem 4.1. Further, we have $\int_{[0,1]^{m}} h(x) d x=C$ for every $h \in \Omega_{2^{n}}$. We can then from Theorem 4.2 conclude that the set $\Lambda$ in addition is invariant $\varphi$-minimal, i.e. given $f \in L^{1}[0,1]$ there exists $g_{*, f} \in \Lambda$ such that

$$
\int_{0}^{1} \varphi\left(g_{*, f}(x)-f(x)\right) d x \leq \int_{0}^{1} \varphi(g(x)-f(x)) d x
$$

for every $g \in \Lambda$ and all convex functions $\varphi: \mathbb{R} \rightarrow \mathbb{R}$.

5.3. The taut string problem in the continuous setting. We now consider an infinite-dimensional analogue of Theorem [2.2, Let $C[0,1]$ and $A C[0,1]$ denote the spaces of continuous respectively absolutely continuous functions on $[0,1]$. We have the following result

Theorem 5.2. Let $F, G \in C[0,1]$ with $F \leq G$. Then the set

$$
\Lambda:=\left\{h \in L^{1}[0,1]: \exists H \in A C[0,1], F \leq H \leq G, h=H^{\prime} \text { a.e. on }[0,1]\right\}
$$

is invariant $K$-minimal with respect to $\left(L^{1}[0,1], L^{\infty}[0,1]\right)$.

Proof. Consider the family of sets $\left\{\Omega_{2^{n}}\right\}_{n \in \mathbb{N}}$ where

$$
\begin{aligned}
& \Omega_{2^{n}}:=\left\{\widehat{h} \in S_{2^{n}}[0,1]: \exists D \in[F(0), G(0)]\right. \text { s.t. } \\
&\left.F\left(\frac{k}{2^{n}}\right) \leq D+\int_{0}^{\frac{k}{2^{n}}} \widehat{h}(s) d s \leq G\left(\frac{k}{2^{n}}\right), k=0,1, \ldots, 2^{n}\right\} .
\end{aligned}
$$

We will now show that $\left\{\Omega_{2^{n}}\right\}_{n \in \mathbb{N}}$ fulfill the conditions of Theorem 4.1 .

It is clear from the definition that $\Omega_{2^{n}}$ is a closed and convex subset of $S_{2^{n}}$. We next show $P_{2^{n}}: \Omega_{2^{n+1}} \rightarrow \Omega_{2^{n}}$. Take $\widehat{h} \in \Omega_{2^{n+1}}$ with corresponding constant $D \in[F(0), G(0)]$ such that

$$
F\left(\frac{k}{2^{n+1}}\right) \leq D+\int_{0}^{\frac{k}{2^{n+1}}} \widehat{h}(s) d s \leq G\left(\frac{k}{2^{n+1}}\right), k=0,1, \ldots, 2^{n+1} .
$$


As

$$
\int_{0}^{\frac{k}{2^{n}}} P_{2^{n}} f(s) d s=\int_{0}^{\frac{k}{2^{n}}} f(s) d s, k=0,1, \ldots, 2^{n},
$$

for any $f \in L^{1}[0,1]$ it follows that

$$
F\left(\frac{k}{2^{n}}\right) \leq D+\int_{0}^{\frac{k}{2^{n}}} P_{2^{n}} \widehat{h}(s) d s \leq G\left(\frac{k}{2^{n}}\right), k=0,1, \ldots, 2^{n},
$$

and therefore $P_{2^{n}} \widehat{h} \in \Omega_{2^{n}}$. So, $P_{2^{n}}: \Omega_{2^{n+1}} \rightarrow \Omega_{2^{n}}$.

The coefficient set $\widetilde{\Omega}_{2^{n}}$ is given by

$$
\begin{aligned}
& \widetilde{\Omega}_{2^{n}}=\left\{c \in \mathbb{R}^{2^{n}}: \exists D \in[F(0), G(0)]\right. \text { s.t. } \\
&\left.F\left(\frac{k}{2^{n}}\right) \leq D+\sum_{i=1}^{k} \frac{c_{i}}{2^{n}} \leq G\left(\frac{k}{2^{n}}\right), k=1, \ldots, 2^{n}\right\} .
\end{aligned}
$$

For $x \in[0,1]$, let

$$
F_{2^{n}}(x):=2^{n}\left(\frac{i_{n}}{2^{n}}-x\right) F\left(\frac{i_{n}-1}{2^{n}}\right)+2^{n}\left(x-\frac{i_{n}-1}{2^{n}}\right) F\left(\frac{i_{n}}{2^{n}}\right)
$$

and

$$
G_{2^{n}}(x):=2^{n}\left(\frac{i_{n}}{2^{n}}-x\right) G\left(\frac{i_{n}-1}{2^{n}}\right)+2^{n}\left(x-\frac{i_{n}-1}{2^{n}}\right) G\left(\frac{i_{n}}{2^{n}}\right) .
$$

So, $F_{2^{n}}$ and $G_{2^{n}}$ are continuous piecewise linear function on $[0,1]$ with nodes in $\frac{k}{2^{n}}$ where $F_{2^{n}}\left(\frac{k}{2^{n}}\right)=F\left(\frac{k}{2^{n}}\right)$ and $G_{2^{n}}\left(\frac{k}{2^{n}}\right)=G\left(\frac{k}{2^{n}}\right), k=0,1, \ldots, 2^{n}$. Let $\Gamma_{F_{2^{n}}, G_{2^{n}}}$ denote the set of all continuous functions piecewise linear functions $f$ on $[0,1]$ with nodes in $\frac{k}{2^{n}}, k=0,1, \ldots, 2^{n}$, and which satisfies the inequalities $F_{2^{n}} \leq f \leq G_{2^{n}}$. Then we see that $\widetilde{\Omega}_{2^{n}}$ can equivalently be expressed as

$$
\widetilde{\Omega}_{2^{n}}=\left\{c \in \mathbb{R}^{2^{n}}: c_{k}=\frac{f\left(\frac{k}{2^{n}}\right)-f\left(\frac{k-1}{2^{n}}\right)}{\frac{1}{2^{n}}}, k=1, \ldots, 2^{n}, f \in \Gamma_{F_{2^{n}}, G_{2^{n}}}\right\} .
$$

Hence, $\widetilde{\Omega}_{2^{n}}$ falls into the category of sets of Theorem 2.2 which gives that it is invariant $K$-minimal with respect to $\left(\ell^{1}, \ell^{\infty}\right)$ on $\mathbb{R}^{2^{n}}$.

From Theorem 4.1 it now follows that the set

$$
\Omega:=\left\{h \in L^{1}[0,1]: P_{2^{n}} h \in \Omega_{2^{n}}, \forall n \in \mathbb{N}\right\}
$$

is invariant $K$-minimal with respect to $\left(L^{1}, L^{\infty}\right)$. The remaining part of the proof is devoted to show the characterization $\Omega=\Lambda$.

We first consider the inclusion $\Lambda \subset \Omega$. Take $h \in \Lambda$ and $n \in \mathbb{N}$. By assumption there exists $H \in A C[0,1]$ such that $h=H^{\prime}$ a.e. on $[0,1]$ with $F \leq H \leq G$. From (46) it follows that $\int_{0}^{\frac{k}{2^{n}}} P_{2^{n}} h(s) d s=\int_{0}^{\frac{k}{2^{n}}} h(s) d s, k=0,1, \ldots, 2^{n}$, and therefore

$$
F\left(\frac{k}{2^{n}}\right) \leq H(0)+\int_{0}^{\frac{k}{2^{n}}} P_{2^{n}} h(s) d s \leq G\left(\frac{k}{2^{n}}\right), k=0,1, \ldots, 2^{n} .
$$

So, $P_{2^{n}} h \in \Omega_{2^{n}}$. As $h \in \Lambda$ and $n \in \mathbb{N}$ were chosen arbitrarily we conclude that $\Lambda \subset \Omega$. 
We now turn to the inclusion $\Lambda \supset \Omega$. Let $h \in \Omega$. Then $P_{2^{n}} h \in \Omega_{2^{n}}$ for every $n \in \mathbb{N}$. It follows that for each $n \in \mathbb{N}$ there exists constant $D_{2^{n}} \in[F(0), G(0)]$ such that

$$
F\left(\frac{k}{2^{n}}\right) \leq D_{2^{n}}+\int_{0}^{\frac{k}{2^{n}}} P_{2^{n}} h(s) d s \leq G\left(\frac{k}{2^{n}}\right)
$$

for $k=0,1, \ldots, 2^{n}$.

Let $y \in[0,1]$ be given. Then $y \in \Gamma_{i_{n}}^{2^{n}}=\left[\frac{i_{n}-1}{2^{n}}, \frac{i_{n}}{2^{n}}\right)$ for some $i_{n} \in\left\{1, \ldots, 2^{n}\right\}$, $n=1,2, \ldots$. From (49) it follows that

$$
F_{2^{n}}(y) \leq D_{2^{n}}+\int_{0}^{y} P_{2^{n}} h(s) d s \leq G_{2^{n}}(y)
$$

as $F_{2^{n}}(x), G_{2^{n}}(x)$ (recall definitions (47) and (48)) and $D_{2^{n}}+\int_{0}^{x} P_{2^{n}} h(s) d s$ interpolate linearly between the nodes $\frac{k}{2^{n}}, k=0,1, \ldots, 2^{n}$. From

$$
\begin{aligned}
& \min \left\{F\left(\frac{i_{n}-1}{2^{n}}\right), F\left(\frac{i_{n}}{2^{n}}\right)\right\} \leq F_{2^{n}}(y) \leq \max \left\{F\left(\frac{i_{n}-1}{2^{n}}\right), F\left(\frac{i_{n}}{2^{n}}\right)\right\}, \\
& \min \left\{G\left(\frac{i_{n}-1}{2^{n}}\right), G\left(\frac{i_{n}}{2^{n}}\right)\right\} \leq G_{2^{n}}(y) \leq \max \left\{G\left(\frac{i_{n}-1}{2^{n}}\right), G\left(\frac{i_{n}}{2^{n}}\right)\right\}
\end{aligned}
$$

and continuity of $F$ and $G$ we obtain

$$
\lim _{n \rightarrow \infty} F_{2^{n}}(y)=F(y)
$$

and

$$
\lim _{n \rightarrow \infty} G_{2^{n}}(y)=G(y) .
$$

Further, $\lim _{n \rightarrow \infty} P_{2^{n}} h=h$ in $L^{1}$ implies that $\lim _{n \rightarrow \infty} \int_{0}^{y} P_{2^{n}} h(s) d s=\int_{0}^{y} h(s) d s$. Moreover, as $\left\{D_{2^{n}}\right\}_{n \in \mathbb{N}}$ is bounded it contains a convergent subsequence $\left\{D_{2^{n_{m}}}\right\}_{m \in \mathbb{N}}$. Let $D:=\lim _{m \rightarrow \infty} D_{2^{n} m}$. As $F(0) \leq D_{2^{n}} \leq G(0)$ it follows that $F(0) \leq D \leq G(0)$. We define

$$
H(y):=\lim _{m \rightarrow \infty}\left(D_{2^{n_{m}}}+\int_{0}^{y} P_{2^{n_{m}}} h(s) d s\right)=D+\int_{0}^{y} h(s) d s .
$$

From (53) it is clear that $H \in A C[0,1]$. Combining (50), (51), (52) and (53) give

$$
F(y) \leq H(y) \leq G(y)
$$

Since $y \in[0,1]$ was chosen arbitrarily we conclude that $F \leq H \leq G$ and therefore $h \in \Lambda$. Further, the choice of $h \in \Omega$ was arbitrary so $\Omega \subset \Lambda$ and the theorem is established.

Remark 5.2. From the proof of Theorem 5.2 we know that the family of sets $\left\{\Omega_{2^{n}}\right\}_{n \in \mathbb{N}}$ defined by (45) satisfy the conditions of Theorem 4.1. If $F(0)=G(0)$ and $F(1)=G(1)$ it follows that $\int_{0}^{1} g(x) d x=F(1)-F(0)$ for every $g \in \Omega_{2^{n}}$. We can then from Theorem 4.2 conclude that the set $\Lambda$ in addition is invariant $\varphi$-minimal, i.e. given $f \in L^{1}[0,1]$ there exists $g_{*, f} \in \Lambda$ such that

$$
\int_{0}^{1} \varphi\left(g_{*, f}(x)-f(x)\right) d x \leq \int_{0}^{1} \varphi(g(x)-f(x)) d x
$$

for every $g \in \Lambda$ and all convex functions $\varphi: \mathbb{R} \rightarrow \mathbb{R}$. 
Remark 5.3. Let $F, G$ be continuous piecewise linear functions on $[0,1]$ with nodes in $\frac{i}{m}, i=0,1, \ldots, m, m \in \mathbb{N}$, where $G\left(\frac{i}{m}\right)=F\left(\frac{i}{m}\right)+\alpha, i=1, \ldots, m-1$, for some $\alpha>0$ and $F(0)=G(0), F(1)=G(1)$. Then the result of Remark 5.2 was known before, see Theorem 4.35 in [19, p. 141].

5.4. Divergence of flows in the continuous setting. Our next result can be seen as an example of one possible infinite-dimensional analogue of divergence of flows on the graph considered in Section 3 . Let $C_{c}^{\infty}\left((0,1)^{m}, \mathbb{R}^{m}\right)$ denote the space of $m$-dimensional smooth vector fields with support in $(0,1)^{m}$. We define $L^{\infty}\left([0,1]^{m}, \mathbb{R}^{m}\right)$ as the space of all $m$-dimensional Lebesgue measurable vector fields $\mathbf{F}=\left(F_{1}, \ldots, F_{m}\right):[0,1]^{m} \rightarrow \mathbb{R}^{m}$ such that $\|\mathbf{F}\|_{L^{\infty}}:=\max _{1 \leq i \leq m}\left\{\left\|F_{i}\right\|_{L^{\infty}}\right\}<\infty$.

Theorem 5.3. Let

$$
M:=\left\{\mathbf{F} \in C_{c}^{\infty}\left((0,1)^{m}, \mathbb{R}^{m}\right):\|\mathbf{F}\|_{L^{\infty}} \leq 1\right\}
$$

and

$$
\operatorname{div} M:=\left\{f \in C_{c}^{\infty}\left((0,1)^{m}\right): f=\operatorname{div} \mathbf{F}, \mathbf{F} \in M\right\} .
$$

Then the closure of $\operatorname{div} M$ in $L^{1}$, denoted $\overline{\operatorname{div} M}$, is an invariant $K$-minimal set with respect to $\left(L^{1}\left([0,1]^{m}\right), L^{\infty}\left([0,1]^{m}\right)\right)$.

Proof. In order to make formulas clear we will consider the case $m=2$. The proof of the case $m>2$ is completely analogous.

Consider the family of sets $\left\{\Omega_{2^{n}}\right\}_{n \in \mathbb{N}}$ given by

$$
\Omega_{2^{n}}:=\left\{g \in S_{2^{n}}\left([0,1]^{2}\right): g=g_{1}+g_{2}, g_{1} \in \Omega_{2^{n}}^{1}, g_{2} \in \Omega_{2^{n}}^{2}\right\}
$$

where

$$
\Omega_{2^{n}}^{1}:=\left\{g_{1} \in S_{2^{n}}\left([0,1]^{2}\right): \sup _{x \in[0,1]}\left|\int_{0}^{x} g_{1}(s, y) d s\right| \leq 1, \int_{0}^{1} g_{1}(s, y) d s=0\right\}
$$

and

$$
\Omega_{2^{n}}^{2}:=\left\{g_{2} \in S_{2^{n}}\left([0,1]^{2}\right): \sup _{y \in[0,1]}\left|\int_{0}^{y} g_{2}(x, t) d t\right| \leq 1, \int_{0}^{1} g_{2}(x, t) d s=0\right\} .
$$

We will now show that $\left\{\Omega_{2^{n}}\right\}_{n \in \mathbb{N}}$ fulfill the conditions of Theorem 4.1 .

Since $\Omega_{2^{n}}^{1}$ and $\Omega_{2^{n}}^{2}$ are bounded, closed and convex subsets of $S_{2^{n}}$ it is clear by construction that $\Omega_{2^{n}}$ also is so. Further, direct computations give that $P_{2^{n}}$ : $\Omega_{2^{n+1}} \rightarrow \Omega_{2^{n}}$

Next, the coefficient set $\widetilde{\Omega}_{2^{n}}$ can be interpreted on a directed graph $(V, E)$ according to the following. Let

$$
V=\left\{v_{i j}: i, j=1, \ldots, 2^{n}\right\}
$$

and

$$
\begin{aligned}
E=\left\{e_{i j}^{h}=\left(v_{i(j+1)}, v_{i j}\right): i=1, \ldots, 2^{n}, j=1, \ldots, 2^{n}-1\right\} \cup \\
\\
\left\{e_{i j}^{v}=\left(v_{(i+1) j}, v_{i j}\right): i=1, \ldots, 2^{n}-1, j=1, \ldots, 2^{n}\right\} .
\end{aligned}
$$

The superscripts $h$ and $v$ stand for "horizontal" respectively "vertical", a notation which is natural when arranging the vertices in a rectangular array. For an example of graph with this structure, see Figure 5. On the edges of the graph we assign 


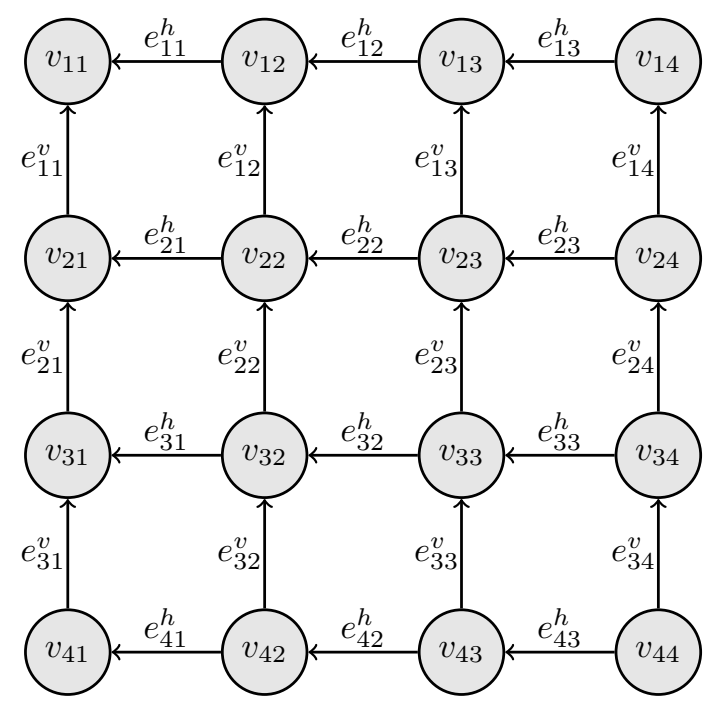

FIGURE 5. Example of graph with $n=2$.

flows according to

$$
f\left(e_{i j}^{h}\right)=\int_{0}^{\frac{j}{2^{n}}} g_{1}\left(s, \frac{i-1}{2^{n}}\right) d s
$$

and

$$
f\left(e_{i j}^{v}\right)=\int_{0}^{\frac{i}{2^{n}}} g_{2}\left(\frac{j-1}{2^{n}}, s\right) d s .
$$

Then $f(e) \in[-1,1]$ for any $e \in E$. Further, recall that the divergence at a vertex $v \in V$, denoted $\operatorname{div} v$, is the sum of all incoming flows minus the sum of all outgoing flows at $v$. Taking into account the structure of $(V, E)$ and (57) and (58) it then follows that

$$
\operatorname{div} v_{i j}=\frac{c_{i j}}{2^{n}}, i, j=1, \ldots, 2^{n}
$$

where $g=\sum_{i, j=1}^{2^{n}} c_{i j} \chi_{\Gamma_{i j}^{2 n}}$. So, the coefficient set $\widetilde{\Omega}_{2^{n}}$ can be interpreted as the set in $S_{V}$ which is the image of the rectangular domain

$$
R_{-1,1}=\left\{f \in S_{E}:-1 \leq f(e) \leq 1, \forall e \in E\right\}
$$

under the divergence operator div $: S_{E} \rightarrow S_{V}$. Recalling Theorem 2.4, we then conclude that $\widetilde{\Omega}_{2^{n}}$ is an invariant $K$-minimal set with respect to $\left(\ell^{1}, \ell^{\infty}\right)$ on $\mathbb{R}^{2^{2 n}}$ (as we consider two-dimensional case $m=2$ ).

We are now in a position where Theorem 4.1 implies that

$$
\Omega:=\left\{h \in L^{1}\left([0,1]^{2}\right): P_{2^{n}} h \in \Omega_{2^{n}}, \forall n \in \mathbb{N}\right\}
$$

is an invariant $K$-minimal set with respect to $\left(L^{1}, L^{\infty}\right)$. The remaining part of the proof is devoted to show the characterization $\Omega=\overline{\operatorname{div} M}$. 
Let us first show the inclusion $\overline{\operatorname{div} M} \subset \Omega$. Take $h \in \overline{\operatorname{div} M}$ and $n \in \mathbb{N}$. Given any $\varepsilon>0$, we can find $h_{\varepsilon} \in \operatorname{div} M$ such that $\left\|h-h_{\varepsilon}\right\|_{L^{1}} \leq \varepsilon$. Now,

$$
h_{\varepsilon}=\operatorname{div} \mathbf{H}_{\varepsilon}=\frac{\partial}{\partial x} H_{\varepsilon, 1}+\frac{\partial}{\partial y} H_{\varepsilon, 2}
$$

for some $\mathbf{H}_{\varepsilon}=\left(H_{\varepsilon, 1}, H_{\varepsilon, 2}\right) \in M$. From the definition of $M$ it is easy to show that $P_{2^{n}} \frac{\partial}{\partial x} H_{\varepsilon, 1} \in \Omega_{2^{n}}^{1}$ and $P_{2^{n}} \frac{\partial}{\partial y} H_{\varepsilon, 2} \in \Omega_{2^{n}}^{2}$. So,

$$
P_{2^{n}} h_{\varepsilon}=P_{2^{n}}\left(\frac{\partial}{\partial x} H_{\varepsilon, 1}+\frac{\partial}{\partial y} H_{\varepsilon, 2}\right)=P_{2^{n}} \frac{\partial}{\partial x} H_{\varepsilon, 1}+P_{2^{n}} \frac{\partial}{\partial y} H_{\varepsilon, 2} \in \Omega_{2^{n}}
$$

From

$$
\left\|P_{2^{n}} h-P_{2^{n}} h_{\varepsilon}\right\|_{L^{1}} \leq\left\|h-h_{\varepsilon}\right\|_{L^{1}} \leq \varepsilon
$$

we then obtain $P_{2^{n}} h \in \Omega_{2^{n}}$ as $\Omega_{2^{n}}$ is a closed subset of $S_{2^{n}}$. Hence, $\overline{\operatorname{div} M} \subset \Omega$ as $h \in \overline{\operatorname{div} M}$ and $n \in \mathbb{N}$ were chosen arbitrarily.

We now show $\overline{\operatorname{div} M} \supset \Omega$. The main problem in this direction is to show $\Omega_{2^{n}} \subset$ $\overline{\operatorname{div} M}$ for every $n \in \mathbb{N}$. For this purpose, we introduce the set

$$
S:=\cup_{n \in \mathbb{N}} \Omega_{2^{n}}
$$

Clearly, $\Omega_{2^{n}} \subset S$ for any $n \in \mathbb{N}$. We introduce further the set

$$
S_{c}:=\left\{f \in S: \exists n \in \mathbb{N} \text { s.t. } f=f_{1}+f_{2}, f_{i} \in \Omega_{2^{n}}^{i}, \operatorname{supp}\left(f_{i}\right) \subset(0,1)^{2}, i=1,2\right\} .
$$

It is important to note in the definition of $S_{c}$ that $\operatorname{supp}\left(f_{i}\right), i=1,2$, is contained in $(0,1)^{2}$ and not just in $[0,1]^{2}$.

Now, we claim $S \subset \overline{S_{c}}$. Let us show this. Take $f \in S$ with decomposition $f=f_{1}+f_{2}, f_{i} \in \Omega_{2^{n}}^{i}, i=1,2$. The basic idea, to be justified below, is that for any $m \in \mathbb{N}, m \geq n$, we can find $g_{i, m} \in \Omega_{2^{m}}^{i}$ with $\operatorname{supp}\left(g_{i, m}\right) \subset(0,1)^{2}$ such that $g_{i, m}$ coincides with $f_{i}$ on the square centered at $(1 / 2,1 / 2)$ with side length $1-\frac{2}{2^{n}}$ and moreover

$$
\lim _{m \rightarrow \infty}\left\|f_{i}-g_{i, m}\right\|_{L^{1}}=0
$$

The properties of $g_{1, m}$ and $g_{2, m}$ imply that $g_{m}:=g_{1, m}+g_{2, m} \in S_{c}$. Further, taking into account (60) we have

$$
\lim _{m \rightarrow \infty}\left\|f-g_{m}\right\|_{L^{1}}=0 .
$$

So, $f \in \overline{S_{c}}$ and since $f \in S$ was chosen arbitrary we conclude that $S \subset \overline{S_{c}}$.

With the basic idea introduced, let us now give the explicit construction of the functions $g_{i, m}$. The approximating function $g_{1, m} \in \Omega_{2^{m}}^{1}$ to $f_{1}$ is constructed according to the following. Put $g_{1, m}(x, y)=0$ if $x \in\left[0, \frac{1}{2^{m}}\right) \cup\left[\frac{2^{m}-1}{2^{m}}, 1\right)$ or $y \in$ $\left[0, \frac{1}{2^{m}}\right) \cup\left[\frac{2^{m}-1}{2^{m}}, 1\right)$. Suppose now $y \in\left[\frac{1}{2^{m}}, \frac{2^{m}-1}{2^{m}}\right)$. Then for $x \in\left[\frac{1}{2^{n}}, \frac{2^{n}-1}{2^{n}}\right)$ we let $g_{1, m}(x, y)=f_{1}(x, y)$ and for $x \in\left[\frac{1}{2^{m}}, \frac{1}{2^{n}}\right) \cup\left[\frac{2^{n}-1}{2^{n}}, \frac{2^{m}-1}{2^{m}}\right)$ we let $g_{1, m}(x, y)=$ $\frac{2^{m} f_{1}(x, y)}{2^{m}-2^{n}}$. The construction of $g_{1, m}$ is now complete and one easily verifies that $g_{1, m} \in \Omega_{2^{m}}^{1}$ and $\operatorname{supp}\left(g_{1, m}\right) \subset(0,1)^{2}$. Further, by the construction of $g_{1, m}$ it follows through direct computations that $\left\|f_{1}-g_{1, m}\right\|_{L^{1}} \rightarrow 0$ as $m \rightarrow \infty$.

An analogous approximating function $g_{2, m} \in \Omega_{2^{m}}^{2}$ to $f_{2}$ is now constructed. We let $g_{2, m}(x, y)=0$ if $x \in\left[0, \frac{1}{2^{m}}\right) \cup\left[\frac{2^{m}-1}{2^{m}}, 1\right)$ or $y \in\left[0, \frac{1}{2^{m}}\right) \cup\left[\frac{2^{m}-1}{2^{m}}, 1\right)$. Suppose $x \in\left[\frac{1}{2^{m}}, \frac{2^{m}-1}{2^{m}}\right)$. Then for $y \in\left[\frac{1}{2^{n}}, \frac{2^{n}-1}{2^{n}}\right)$ we let $g_{2, m}(x, y)=f_{2}(x, y)$ and for 
$y \in\left[\frac{1}{2^{m}}, \frac{1}{2^{n}}\right) \cup\left[\frac{2^{n}-1}{2^{n}}, \frac{2^{m}-1}{2^{m}}\right)$ we let $g_{2, m}(x, y)=\frac{2^{m} f_{2}(x, y)}{2^{m}-2^{n}}$. From construction it follows that $g_{2, m} \in \Omega_{2^{m}}^{2}, \operatorname{supp}\left(g_{2, m}\right) \subset(0,1)^{2}$ and $\left\|f_{2}-g_{2, m}\right\|_{L^{1}} \rightarrow 0$ as $m \rightarrow \infty$.

With $S \subset \overline{S_{c}}$ established, the next step is to show that $\overline{S_{c}} \subset \overline{\operatorname{div} M}$. Let $f \in$ $S_{c}$ be given. We then have a decomposition $f=f_{1}+f_{2}$ where $f_{i} \in \Omega_{2^{n}}^{i}$ and $\operatorname{supp}\left(f_{i}\right) \subset(0,1)^{2}, i=1,2$, for some $n \in \mathbb{N}$ according to (59). Now, let $\mathbf{F}=$ $\left(F_{1}, F_{2}\right) \in C_{c}\left((0,1)^{2}, \mathbb{R}^{2}\right)$ denote the vector field where

$$
F_{1}(x, y):=\int_{0}^{x} f_{1}(s, y) d s
$$

and

$$
F_{2}(x, y):=\int_{0}^{y} f_{2}(x, t) d t
$$

From the definition it follows that $\|\mathbf{F}\|_{L^{\infty}} \leq 1$. We will next modify $\mathbf{F}$ to a smooth vector field belonging to $M$.

Let $\left\{\rho_{m}\right\}_{m \in \mathbb{N}}$ be a sequence of mollifiers on $\mathbb{R}^{2}$, i.e. $\rho_{m} \in C_{c}^{\infty}\left(\mathbb{R}^{2}\right), \operatorname{supp}\left(\rho_{m}\right) \subset$ $\overline{B\left(0, \frac{1}{m}\right)}$ where $B\left(0, \frac{1}{m}\right)$ denotes the Euclidean ball in $\mathbb{R}^{2}$ centered at 0 with radius $\frac{1}{m}, \int_{\mathbb{R}^{2}} \rho_{m}(x) d x=1$ and $\rho_{m} \geq 0$. By recalling some standard facts regarding convolution, see e.g. 3. Propositions 4.18 and 4.20, pp. 106-107], we conclude that $F_{i} * \rho_{m} \in C_{c}^{\infty}\left((0,1)^{2}\right), i=1,2$, for $m$ large enough. Moreover, $\left\|F_{i} * \rho_{m}\right\|_{L^{\infty}} \leq$ $\left\|F_{i}\right\|_{L^{\infty}}\left\|\rho_{m}\right\|_{L^{1}}=\left\|F_{i}\right\|_{L^{\infty}} \leq 1$. So for $m$ large enough we have

$$
\mathbf{F}_{m}:=\left(F_{1} * \rho_{m}, F_{2} * \rho_{m}\right) \in M
$$

From (61) it follows that the piecewise constant function $f_{1}(x, y)$ is the partial derivative with respect to $x$ of $F_{1}(x, y)$ on $(0,1)^{2}$ except for the lines $x=\frac{i}{2^{n}}$, $i=1, \ldots, 2^{n}-1$. We therefore have for any $(a, b) \in(0,1)^{2}$

$$
\begin{aligned}
& \frac{\partial}{\partial x}\left(F_{1} * \rho_{m}\right)(a, b)=\left(F_{1} * \frac{\partial \rho_{m}}{\partial x}\right)(a, b)=\int_{0}^{1} \int_{0}^{1} F_{1}(u, v) \frac{\partial \rho_{m}}{\partial x}(a-u, b-v) d u d v \\
& =\int_{0}^{1} \sum_{i=1}^{2^{n}} \int_{\frac{i-1}{2^{n}}}^{\frac{i}{2^{n}}} F_{1}(u, v) \frac{\partial \rho_{m}}{\partial x}(a-u, b-v) d u d v \\
& =\int_{0}^{1} \sum_{i=1}^{2^{n}}\left(\left[-F_{1}(u, v) \rho_{m}(a-u, b-v)\right]_{\frac{i-1}{2^{n}}}^{\frac{i}{2^{n}}}+\int_{\frac{i-1}{2^{n}}}^{\frac{i}{2^{n}}} f_{1}(u, v) \rho_{m}(a-u, b-v) d u\right) d v \\
& =\int_{0}^{1} \sum_{i=1}^{2^{n}} \int_{\frac{i-1}{2^{n}}}^{\frac{i}{2^{n}}} f_{1}(u, v) \rho_{m}(a-u, b-v) d u d v \\
& =\int_{0}^{1} \int_{0}^{1} f_{1}(u, v) \rho_{m}(a-u, b-v) d u d v=\left(f_{1} * \rho_{m}\right)(a, b) .
\end{aligned}
$$


When going from the third to the fourth line in the computation above we use

$$
\begin{aligned}
& \sum_{i=1}^{2^{n}}\left[-F_{1}(u, v) \rho_{m}(a-u, b-v)\right]_{u=\frac{i-1}{2^{n}}}^{\frac{i}{2^{n}}}= \\
& =F_{1}\left(\frac{1}{2^{n}}, v\right) \rho_{m}\left(a-\frac{1}{2^{n}}, b-v\right)-F_{1}\left(\frac{2}{2^{n}}, v\right) \rho_{m}\left(a-\frac{2}{2^{n}}, b-v\right)+ \\
& F_{1}\left(\frac{2}{2^{n}}, v\right) \rho_{m}\left(a-\frac{2}{2^{n}}, b-v\right)+\ldots-F_{1}\left(\frac{2^{n}-1}{2^{n}}, v\right) \rho_{m}\left(a-\frac{2^{n}-1}{2^{n}}, b-v\right)+ \\
& F_{1}\left(\frac{2^{n}-1}{2^{n}}, v\right) \rho_{m}\left(a-\frac{2^{n}-1}{2^{n}}, b-v\right)-F_{1}\left(\frac{2^{n}}{2^{n}}, v\right) \rho_{m}\left(a-\frac{2^{n}}{2^{n}}, b-v\right)= \\
& =F_{1}\left(\frac{1}{2^{n}}, v\right) \rho_{m}\left(a-\frac{1}{2^{n}}, b-v\right)-F_{1}(1, v) \rho_{m}(a-1, b-v)=0 .
\end{aligned}
$$

Let us show why the last equality holds in the computations above. As $F_{1}(u, v)=$ $\int_{0}^{u} f_{1}(s, v) d s$ (recall (61) ) where $\operatorname{supp}\left(f_{1}\right) \in(0,1)^{2}$ and $f_{1} \in \Omega_{2^{n}}^{1}$ (recall definition (59) of $S_{c}$ and definition (55) of $\left.\Omega_{2^{n}}^{1}\right)$ we have $F_{1}\left(\frac{1}{2^{n}}, v\right)=0$. Further, $f_{1} \in \Omega_{2^{n}}^{1}$ implies $F_{1}(1, v)=0$.

With analogous arguments and computations we can show

$$
\frac{\partial}{\partial y}\left(F_{2} * \rho_{m}\right)=f_{2} * \rho_{m}
$$

So,

$$
\operatorname{div} \mathbf{F}_{m}=f_{1} * \rho_{m}+f_{2} * \rho_{m} .
$$

From $f_{i} * \rho_{m} \stackrel{L^{1}}{\rightarrow} f_{i}, i=1,2$, as $m \rightarrow \infty$, see [3, Theorem 4.22, p. 109], we obtain

$$
\operatorname{div} \mathbf{F}_{m} \stackrel{L^{1}}{\rightarrow} f_{1}+f_{2}=f
$$

as $m \rightarrow \infty$. From $\mathbf{F}_{m} \in M$, for large $m$, it then follows that $f \in \overline{\operatorname{div} M}$. As $f \in S_{c}$ was arbitrary we obtain $\overline{S_{c}} \subset \overline{\operatorname{div} M}$. To sum up, the following inclusions

$$
\Omega_{2^{n}} \subset S \subset \overline{S_{c}} \subset \overline{\operatorname{div} M}
$$

hold for every $n \in \mathbb{N}$.

Take now $h \in \Omega$. Since $\Omega_{2^{n}} \subset \overline{\operatorname{div} M}$ for every $n \in \mathbb{N}$ and $P_{2^{n}} h \stackrel{L^{1}}{\rightarrow} h$ as $n \rightarrow \infty$ we must have $h \in \overline{\operatorname{div} M}$. So, $\Omega \subset \overline{\operatorname{div} M}$ which completes the proof.

Remark 5.4. From the proof of Theorem 5.3 we know that the family of sets $\left\{\Omega_{2^{n}}\right\}_{n \in \mathbb{N}}$ defined by 54) satisfy the conditions of Theorem 4.1. Further, 54, (55) and (56) imply that $\int_{[0,1]^{m}} g(x) d x=0$ for every $g \in \Omega_{2^{n}}$. We can then from Theorem 4.2 conclude that $\overline{\operatorname{div} M}$ in addition is invariant $\varphi$-minimal, i.e. given $f \in L^{1}\left([0,1]^{m}\right)$ there is an element $g_{*, f} \in \overline{\operatorname{div} M}$ such that

$$
\int_{[0,1]^{m}} \varphi\left(g_{*, f}(x)-f(x)\right) d x \leq \int_{[0,1]^{m}} \varphi(g(x)-f(x)) d x
$$

holds for every $g \in \overline{\operatorname{div} M}$ and all convex functions $\varphi: \mathbb{R} \rightarrow \mathbb{R}$. In [19], the possibility to have such result under some additional conditions was pointed out, see Remark 4.47 on p. 150. 


\section{REFERENCES}

[1] R. E. Barlow, D. J. Bartholomew, J. M. Bremner, and H. D. Brunk. Statistical Inference under Order Restrictions: The Theory and Application of Isotonic Regression. Wiley Series in Probability and Mathematical Statistics. John Wiley \& Sons, London, 1972.

[2] C. Bennett and R. Sharpley. Interpolation of Operators. Pure and Applied Mathematics, Vol. 129. Academic Press, Boston, MA, 1988.

[3] H. Brezis. Functional Analysis, Sobolev Spaces and Partial Differential Equations. Universitext. Springer, New York, 2011.

[4] Yu. A. Brudnyi and N. Ya. Krugljak. Interpolation Functors and Interpolation Spaces, vol. 1. North-Holland Mathematical Library, Vol. 47. North-Holland, Amsterdam, 1991.

[5] A. P. Calderón. Spaces between $L^{1}$ and $L^{\infty}$ and the theorem of Marcinkiewicz. Studia Math., 26:273-299, 1966.

[6] K.-M. Chong. Some extensions of a theorem of Hardy, Littlewood and Pólya and their applications. Canad. J. Math., 26:1321-1340, 1974.

[7] G. B. Dantzig. A control problem of Bellman. Management Sci., 17:542-546, 1971.

[8] N. Dunford and J. T. Schwartz. Linear Operators. I. General Theory. Pure and Applied Mathematics, Vol. 7. Interscience Publishers, Inc., New York, 1958.

[9] G. H. Hardy, J. E. Littlewood, and G. Pólya. Some simple inequalities satisfied by convex functions. Messenger of Mathematics, 58:145-152, 1929.

[10] R. A. Horn and C. R. Johnson. Matrix Analysis. Cambridge University Press, Cambridge, second edition, 2013.

[11] F. K. Hwang and U. G. Rothblum. Partition-optimization with Schur convex sum objective functions. SIAM J. Discrete Math., 18:512-524, 2005.

[12] N. Kruglyak and E. Setterqvist. Discrete taut strings and real interpolation. J. Funct. Anal., 270:671-704, 2016

[13] M. Lifshits and E. Setterqvist. Energy of taut strings accompanying Wiener process. Stoch. Proc. Appl., 125:401-427, 2015.

[14] W. A. J. Luxemburg. Rearrangement-invariant Banach function spaces. Queen's Papers in Pure and Appl. Math., 10:83-144, 1967.

[15] E. Mammen and S. van de Geer. Locally adaptive regression splines. Ann. Statist., 25:387$413,1997$.

[16] Y. Meyer. Oscillating Patterns in Image processing and Nonlinear Evolution Equations. University Lecture Series, 22. American Mathematical Society, Providence, R.I., 2001.

[17] J. Niyobuhungiro and E. Setterqvist. A new reiterative algorithm for the Rudin-Osher-Fatemi denoising model on the graph. In Proceedings of the 2nd International Conference on Intelligent Systems and Image Processing 2014, pages 81-88, September 2014. See http://liu.divaportal.org/smash/get/diva2:719719/FULLTEXT01.pdf for full version.

[18] J. D. Salehi, Z.-L. Zhang, J. Kurose, and D. Towsley. Supporting stored video: Reducing rate variability and end-to-end resource requirements through optimal smoothing. IEEE/ACM Trans. Networking, 6:397-410, 1998.

[19] O. Scherzer, M. Grasmair, H. Grossauer, M. Haltmeier, and F. Lenzen. Variational Methods in Imaging. Applied Mathematical Sciences, 167. Springer, New York, 2009.

[20] E. Setterqvist and R. Forchheimer. Real-time communication systems based on taut strings. Submitted.

[21] A. Veinott. Least d-majorized network flows with inventory and statistical applications. Management Sci., 17:547-567, 1971.

Department of Mathematics, Linköping University, SE-581 83 LinköPing, Sweden E-mail address: natan.kruglyak@liu.se

Department of Mathematics, LinkÖPing University, SE-581 83 LinköPing, Sweden E-mail address: eric.setterqvist@liu.se 